This is the final peer-reviewed accepted manuscript of:

Formentin, S.M., Zanuttigh, B.

A Genetic Programming based formula for wave overtopping by crown walls and bullnoses

(2019) Coastal Engineering, 152, art. no. 103529

the final published version is available online at:

https://doi.org/10.1016/i.coastaleng.2019.103529

(02019. This manuscript version is made available under the Creative Commons AttributionNonCommercial-NoDerivs (CC BY-NC-ND) 4.0 International License

(http://creativecommons.org/licenses/by-nc-nd/4.0/) 


\title{
A Genetic Programming based formula for wave overtopping by crown walls and bullnoses
}

\author{
Sara Mizar Formentin'1, Barbara Zanuttigh
}

1) University of Bologna, DICAM, Viale Risorgimento 2, 40136 Bologna, Italy, saramizar.formentin2@unibo.it, barbara.zanuttigh@unibo.it

\begin{abstract}
The purpose of this contribution is to propose a new method for the parametrization of the reductive effects induced by crown walls and bullnoses on the average wave overtopping discharge $(q)$ at coastal structures. The method consists of a formula for calculating an influence factor $V_{G P}^{*}$ to account for the single or combined effects of the structural elements. The formula for $V_{G P}^{*}$ is conceived to be included in the $q$ formula by EurOtop (2018). The new formula was developed on the basis of the Genetic Programming (GP) technique trained on a database of nearly 1000 data on wave overtopping at dikes with berms or promenades, crown walls and bullnoses. Part of the data are derived from new experiments carried out by the authors to extend the experience available from the literature and create a database of structure configurations sufficiently wide and appropriately assorted to be used for training the GP. The rough formula for predicting $Y_{G P}^{*}$ obtained by the pure application of the GP was optimized to achieve a greater accuracy in the representation of both the breaking and non-breaking wave conditions. The estimations of $q$ obtained with the new influence factor $V_{G P}^{*}$ are physically meaningful and satisfactory accurate, and overcome the underestimation bias affecting the predictions from the available formulae.
\end{abstract}

Keywords: crown walls; bullnoses; wave overtopping; genetic programming; experimental data 


\section{Highlights}

A new formula representing the reducing effects of crown walls and bullnoses on the overtopping discharge is proposed

The formula is developed based on Genetic Programming fitting up to 1000 new and existing data

The formula applies to smooth structures under breaking and non-breaking waves

Different combinations of structural elements can be represented by means of the same formulation

The formula provides accurate, conservative and physically meaningful estimates of the overtopping discharge 


\section{Introduction}

The intensification of storm events, in combination with the sea level rise, exposes the traditional costal protections, such as dikes and seawalls, to increasing wave loads and overtopping rates. Several measures and solutions to mitigate coastal risks have been investigated (inter alia, Touili et al., 2014; Zanuttigh et al., 2014). One of the engineering-based solutions consists in the upgrade of existing defense structures by adding structural elements such berms, armour and crest units, crown walls, etc. (Burcharth et al., 2014). The addition of crown walls or the inclusion of bullnoses and parapets represent economic and aesthetic-compatible solutions to effectively reduce the overtopping discharges $(q)$, and their employment is increasing rapidly.

Up to the 2010's, the literature studies on the effects of wave walls, parapets and bullnoses on the reduction of $q$ are few and relatively fragmented (EurOtop, 2007). Among these, it is worthy to mention the earliest experiments on recurved parapets on vertical and sloping seawalls carried out by Owen and Steele (1991), the study by Kortenhaus et al. (2001) on the influence of overhanging deflectors on top of vertical walls or steep embankments and the experience collected within the FP7 EU-project CLASH on oblique and recurved parapets (Kortenhaus et al., 2003). The first systematic work on this topic is represented by the campaign of experimental investigations conducted by Van Doorslaer et al. (2015), VD, hereinafter. The study examined the typical defense structures of the Belgian coasts, characterized by smooth dikes, with a long and mildly sloping promenade above the still water level. The authors hypothesized to upgrade the existing structures by constructing a crown wall directly on the slopes or at the end of the promenades, including or not a bullnose, bn hereinafter. VD combined different geometries, collecting more than 1,000 experiments on wave overtopping. The study prompted the first organic set of formulae parametrizing the combined effect of wall, bn, and promenades into a reduction coefficient $\gamma^{*}$ to be included in the EurOtop (2007) equations for the prediction of $q$ (TAW, 2002). The expressions for $\gamma^{*}$ vary according to the combination of structure elements and their validity is limited to non-breaking wave conditions exclusively. Based on the results by Van Doorslaer et al. (2016), the coefficient $V^{*}$ was adopted in the updated version of the EurOtop (2018) manual and applied to the new formula by Van der Meer and Bruce (2014) for the prediction of $q$, which replaces EurOtop (2007).

Recently, Zanuttigh and Formentin (2018), on the basis of a new set of experimental and numerical investigations, proposed a correction to the factor $V^{*}$ in order to extend the work by VD to breaking wave conditions and to more conventional structure types, such as dikes with berms of limited width.

Both EurOtop (2018) and Zanuttigh and Formentin (2018) showed how the use of $V^{*}$ apparently provides a physically-coherent representation of the wave overtopping at structures with walls and bullnoses, bns hereinafter. Both the works are based on the assumption that the original $V^{*}$ factor conceived by VD for EurOtop (2007) could be straightforwardly applied to the EurOtop (2018) version of the equations for $q$, but never checked the consistency among predictions and measured values of $q$.

The present study starts with the revision and verification of the original and updated formulae for $V^{*}$, by re-applying the two methods to new and existing data. By comparing predictions and measurements of $q$, it was found that both the methods significantly underestimate the $q$-values. 
The aim of this work is to present a completely new formula for the parametrization of the effects of crown walls and bns, which can ensure accurate and cautious estimates of $q$ and, at the same time, which is of simple and straightforward application. The new method, which is meant to be directly applicable to the EurOtop (2018) formulae, was developed based on the Genetic Programming (GP). This innovative technique belongs to the field of the Artificial Intelligence and was already and successfully applied in Coastal Engineering for the prediction of the scour at the trunk section of breakwaters (Pourzangbar et al., 2017) and of the wave run-up (Power et al., 2019). Differently from more conventional machine-learning tools, such as neural networks, the predicting tools delivered by GP are algebraic formulae - and not black-boxes - which offer the tool developer the possibility of a direct interaction. Furthermore, the derived formula can be updated to fit new data. The learning phase of GP is extremely quick (a few minutes) and the computational effort significantly low, even in case of large amounts of data. GP is applied to the representation of the wave overtopping for the first time in this contribution.

The paper is organized as follows. Section 2 presents the new experimental and numerical database of wave overtopping experiments at dikes with berms, crown walls and bns conducted by the authors and used to verify the existing methods and calibrate the new one. The literature overview and the results of the application of the existing methods to the prediction of the overtopping discharges are given in Section 3. Section 4 firstly introduces the reader to the basic elements of the GP technique, and it then illustrates the scheme and the parameters adopted to apply the GP to the modelling of the effects of crown walls and bns. Finally, the Section presents the new formula for the prediction of the coefficient $V^{*}$, which was obtained by the application of the GP technique and conveniently adjusted to fit all the available data and to offer a simpler and more physically-based expression. The application of the GP-based formula to the available data is presented in Section 5 . The conclusions of the work are finally drawn in Section 6.

\section{The new database}

A new campaign of laboratory experiments and numerical modelling on wave overtopping at structures with crown walls and bns was conducted. The campaign was aimed at extending the experience collected by VD considering a different structure type under breaking and nonbreaking waves. For all the tested conditions, the wave breaking/non-breaking is supposed to occur for the combination of wave steepness and structure slope, i.e. in relation to the values of $\xi_{m-1,0}$.

The setup of the new tests was based on the numerical database on wave overtopping at dikes collected by Formentin et al. (2014) and recently updated by Formentin and Zanuttigh (2018a). These works were in turn inspired to the experiments on dikes carried out in 1:10 scale by Schüttrumpf and Oumeraci (2005) in the small wave flume of the Technical University of Braunschweig. The same scale 1:10 was adopted within the numerical investigations by Formentin and Zanuttigh (2018a) and kept for the new campaign of numerical modelling. The new numerical tests were setup by replicating the structure configurations modeled by Formentin and Zanuttigh (2018a) and including the crown walls and bns on top of the dikes. The size and 
the characteristics of the walls were set up following the work by Van Doorslaer et al. (2015) and rescaled to $1: 10$ to fit the original dikes size.

Contemporary to the numerical modelling, new experimental tests were carried out in the small wave flume of the laboratory of Bologna. The purpose of these tests was to validate and extend the numerical tests. Due to the lab size and constraints, the laboratory experiments were conducted in 1:20 scale (i.e. 1:2 scale with respect to the numerical modelling dataset).

It is worthy to stress that, similarly to VD, the new numerical and lab experiments aim at representing "realistic" cases at prototype scale, but they do not refer to any existing real structure or specific wave climate.

The tested geometries and the scheme adopted for their representation are illustrated in Subsection 2.1. The setup and the details about the tested configurations within the numerical modelling and the laboratory experiments are provided in the Sub-sections 2.1 and 2.2, respectively. The results of the numerical code are checked and validated against new and existing experiments in Sub-section 2.3. All the measures reported in the manuscript refer to the model scales, if not differently specified.

\subsection{Tested configurations}

The typical cross-section of the structures tested within the numerical code and in the laboratory is provided in Figure 1. The symbols and the parameters adopted to describe the geometry in Figure 1, and used in this contribution, follow the structure schematization of the EurOtop (2018) manual and database (Zanuttigh et al., 2016; Formentin et al., 2017).

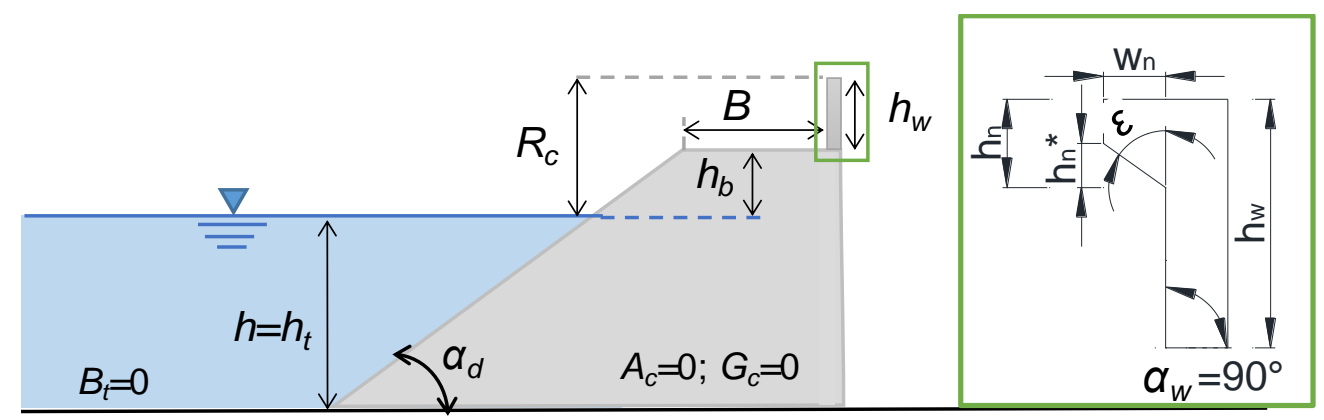

Figure 1- Scheme of the tested cross-sections (from Zanuttigh and Formentin, 2018).

All the new tested cross-sections consist of smooth dike berms and a crown wall at the inshore edge of the berm width $(B)$. In part of the tests, a bn was included at the top of the crown wall. The geometry of the bn is defined by the two parameters $\lambda$ and $\varepsilon$, which represent, respectively, the ratio between the bn and the crown wall height, $\left(\lambda=h_{n} / h_{w}\right)$ and the inclination of the bn; $\lambda=0$ and $\varepsilon=0$ mean no bn, while $\varepsilon>0$ if the bn is seaward inclined. The berm was in emerged conditions $\left(h_{b}<0\right)$ or in correspondence of the still water level $\left(h_{b}=0\right)$, and always included in the run-up/down 
area. The study by VD involved instead the typical cross-sections of the Belgian coasts, where the berms are rather promenades, positioned above the still water level, mildly sloping and characterized by significantly longer values of $B$.

Both the promenades investigated by VD and the new cross-sections tested at the University of Bologna (UB tests, hereinafter) were schematized as smooth berms. This choice was made to be consistent with the EurOtop (2018) representation of similar tests and across the EurOtop database. More details about this schematization are given in Zanuttigh and Formentin (2018).

Table 1 summarizes the main parameters of the new numerical and experimental database, in comparison with the experiments carried out by VD. The shaded cells in this Table remark the ranges of the parameters which extend the field of investigation by VD. The symbols adopted in Table 1 refer to the scheme of Figure 1. All the measures in Table 1 are rescaled to prototype conditions to allow the direct comparison among the new tests (scales 1:10 and 1:20 for numerical and experimental tests, respectively) and VD (scale 1:25).

Table 1 - Tested configurations within the numerical model and in the laboratory compared to the tests by VD. The range of parameters of the new tests which extend the work by VD are marked by green-shading. All the measures are at prototype scale.

\begin{tabular}{|c|c|c|c|c|}
\hline Parameter & Description of the parameter & $\begin{array}{l}\text { Numerical } \\
\text { model }\end{array}$ & $\begin{array}{l}\text { Laboratory } \\
\text { experiments }\end{array}$ & VD \\
\hline $\boldsymbol{h}_{w}[\mathrm{~m}]$ & Crown wall height & $1.5 ; 2.0$ & $0.8 ; 1.0$ & $0.5 ; 1 ; 1.5 ; 2$ \\
\hline $\boldsymbol{R}_{c}[\mathrm{~m}]$ & $\begin{array}{l}\text { Emergence of the crown wall with } \\
\text { respect to the swl }\end{array}$ & $2.0 ; 2.5 ; 3.0$ & {$[0.8 ; 1.6]$} & {$[3.0 ; 6.75]$} \\
\hline $\boldsymbol{h}_{b}[\mathrm{~m}]$ & $\begin{array}{l}\text { Emergence of the berm with respect } \\
\text { to the swl }\end{array}$ & $-1.0 ; 0$ & $-0.6 ;-0.5 ; 0$ & {$[-6.0 ; 0.75]$} \\
\hline $\boldsymbol{B}[\mathrm{m}]$ & Berm width & $3.0 ; 6.0 ; 9.0$ & $3 ; 6$ & $0 ; 8.25 ; 16.5 ; 25$ \\
\hline $\boldsymbol{h}[\mathrm{m}]$ & Water depth in front of the structure & $7.5 ; 8.5$ & {$[6.4 ; 7]$} & {$[8.75 ; 12.25]$} \\
\hline $\boldsymbol{H}_{\boldsymbol{s}}[\mathrm{m}]$ & Target wave height & 2.0 & $1.0 ; 1.2$ & {$[1.75 ; 5.25]$} \\
\hline$H_{s} / L_{m-1,0}[-]$ & Target wave steepness & $0.02 ; 0.03 ; 0.04$ & $0.03 ; 0.04$ & {$[0.01 ; 0.05]$} \\
\hline$\xi_{m-1,0}[-]$ & $\begin{array}{l}\text { Breaker parameter based on the } \\
\text { spectral wave period }\end{array}$ & {$[1.28 ; 4.26]$} & {$[1.20 ; 3.28]$} & {$[2.15 ; 4.90]$} \\
\hline $\boldsymbol{R}_{c} / \boldsymbol{H}_{s}[-]$ & $\begin{array}{l}\text { Relative structure freeboard } \\
\text { considering the crown wall }\end{array}$ & $1 ; 1.25 ; 1.5$ & {$[0.67 ; 1.5]$} & {$[0.83 ; 3.15]$} \\
\hline $\boldsymbol{h}_{w} / \boldsymbol{R}_{c}[-]$ & $\begin{array}{l}\text { Ratio between the crown wall height } \\
\text { and the structure freeboard }\end{array}$ & $0.6 ; 0.67 ; 1$ & {$[0.53 ; 1.22]$} & {$[0.07 ; 0.89]$} \\
\hline$h_{b} / H_{s}[-]$ & $\begin{array}{l}\text { Relative berm emergence with } \\
\text { respect to the swl }\end{array}$ & $-0.5 ; 0$ & $-0.5 ; 0$ & {$[-2.2 ; 0.4]$} \\
\hline$B / L_{m-1,0}[-]$ & Relative berm width width & {$[0.013 ; 0.102]$} & {$[0.055 ; 0.238]$} & {$[0 ; 0.4]$} \\
\hline $\cot \left(\alpha_{d}\right)[-]$ & $\begin{array}{c}\text { Cotangent of the off-shore structure } \\
\text { slope }\end{array}$ & $2 ; 3 ; 4$ & $2 ; 4$ & $2 ; 3$ \\
\hline$\lambda[-]$ & $\begin{array}{l}\text { Ratio between the bn and the crown } \\
\text { wall height, } h_{n} / h_{w}(\lambda=0 \text { means no bn) }\end{array}$ & $0 ; 0.25 ; 0.30 ; 0.375$ & $0 ; 0.375$ & $\begin{array}{c}0 ; 0.25 ; 0.30 \\
0.375 ; 0.5\end{array}$ \\
\hline$\varepsilon\left[^{\circ}\right]$ & Bn inclination $\left(\varepsilon=0^{\circ}\right.$ means no bn) & $0 ; 30 ; 45 ; 60$ & $0 ; 30$ & $0 ; 15 ; 30 ; 45 ; 60$ \\
\hline$\#$ & Number of tested configurations & 91 & 118 & 596 \\
\hline
\end{tabular}




\subsection{The numerical tests}

The numerical simulations were run with the IH2VOF code developed by the University of Cantabria (Lara et al., 2011) and recently modified by Formentin and Zanuttigh (2018a) to represent the wave overtopping process also in case of structures characterized by a "dry landward area", i.e. without the presence of the water in-shore (as in the scheme of Figure 1). The IH2VOF code solves the Reynolds Average Navier-Stokes (RANS) equations, decomposing the instantaneous fields of the flow velocity and pressure in to average and turbulent components, and tracks the free surface with the Volume of Fluid (VOF) method. The turbulent kinetic energy (k) and the turbulent dissipation rate $(\varepsilon)$ are modelled through the $k-\varepsilon$ equations (Rodi, 1980; Lin and Liu, while the influence of turbulence fluctuations on the mean flow field is represented by the Reynolds stresses. The full description and the rigorous mathematical formulation of the equation of the IH2VOF code are given in Liu et al. (1999) and Hsu et al. (2002).

The numerical tests were carried in 1:10 scale to reproduce exactly the scale of the experimental tests by Schüttrumpf and Oumeraci (2005) for smooth dikes. The model was indeed already calibrated and validated for these cases (Formentin and Zanuttigh, 2018a) and these new numerical tests were built on the existing dataset to analyze specifically the effects of crown walls and bullnoses on the wave overtopping discharge.

Figure 2 illustrates the computational domain set up to run all the simulations. It was $41 \mathrm{~m}$ long and $2 \mathrm{~m}$ high, and it was discretized with a structured mesh of rectangles. The mesh resolution in the vertical direction ( $\mathrm{z}$ ) was constant and equal to $0.01 \mathrm{~m}$, while a variable mesh was used for the cross-shore direction ( $x$ ). The highest resolution of $\Delta x=0.01 \mathrm{~m}$ was used around the crown wall and in the run-up area, and it was made linearly decreasing towards the left and the right boundary sections up to approximately $\Delta x=0.04 \mathrm{~m}$. In total, the mesh included 1733 and 201 cells in the $x$-direction and the $z$-direction respectively. The use of the modified version of the code by Formentin and Zanuttigh (2018a) allowed the storage of the overtopping volumes in a numerical reservoir placed on-shore the structures (see Figure 2) and in turn the derivation of the average overtopping discharges from the volumes.

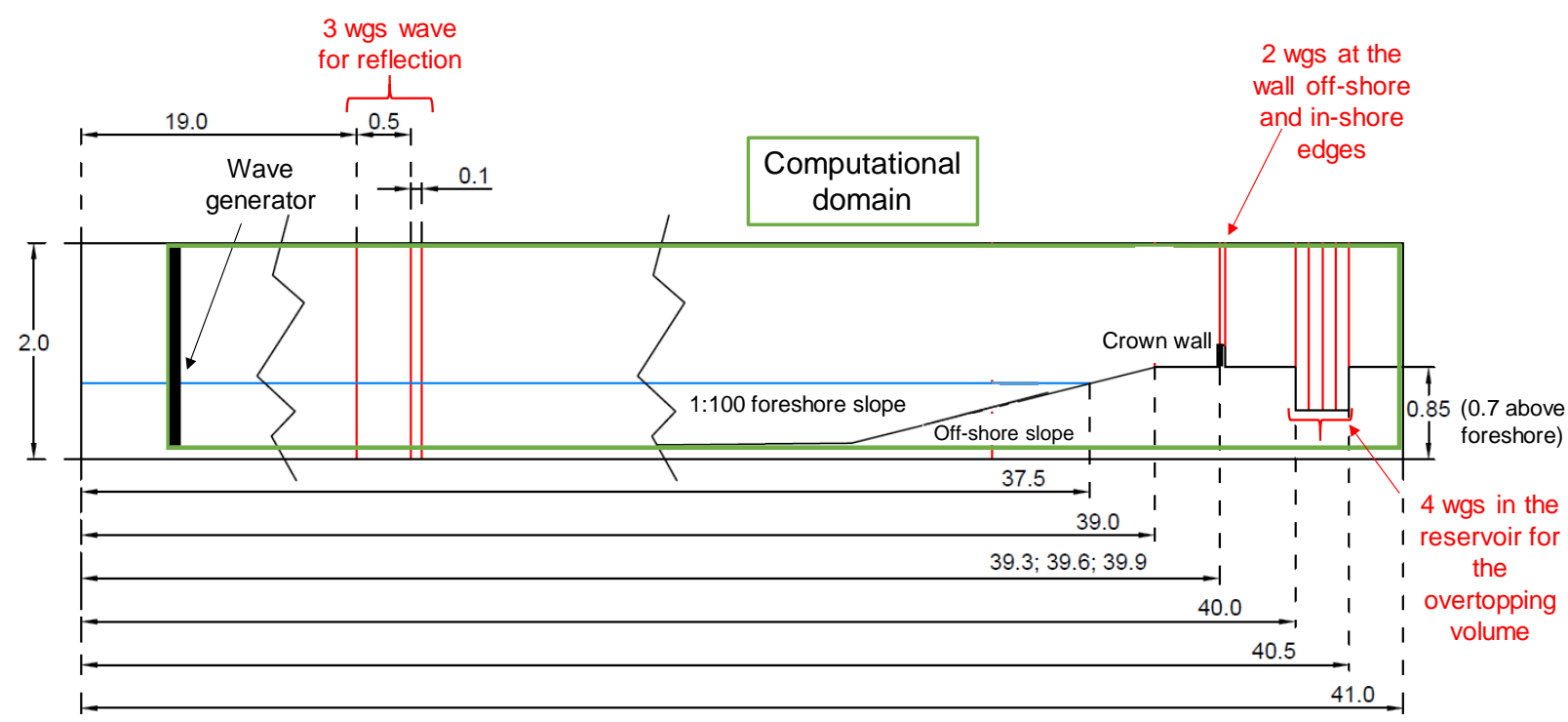

Figure 2 - Layout of the numerical flume and wage gauges. All the measures are in $\mathrm{m}$. 
The basic dike configurations investigated by Formentin and Zanuttigh (2018a) were modified by considering different berm widths $B(0.3,0.6,0.9 \mathrm{~m})$ and off-shore slopes $\cot \left(\alpha_{d}\right)(2,3,4)$ and by including the crown walls, with and without bn, situated at the berm on-shore edge. The geometrical features of the crown walls and of the bns, i.e. the crown wall heights $h_{w}(0.15$ and $0.2 \mathrm{~m})$, the ratios $\lambda$ between the bn height and the wall height $(0.25 ; 0.3 ; 0.375)$, the inclinations $\varepsilon$ of the bn $\left(30,45,60^{\circ}\right)$ and the berm relative emergence $h_{b} / H_{s}(-1.5$ and 0$)$ were defined based on the values or ranges already investigated by VD.

Each simulation lasted $750 \mathrm{~s}$, leading to a number of tested irregular waves between 400 and 500. Each wave was characterized by a Jonswap spectrum with a peak enhancement factor $\gamma=3.3$. One target wave height $H_{s}=0.2 \mathrm{~m}$ and different peak wave periods $T_{p}$ were selected in order to realize both breaking and non-breaking conditions and to determine target wave steepnesses $H_{s} / L_{m-1,0}$ in the range $[0.02 ; 0.04]$, where $L_{m-1,0}$ is the wave length computed from the spectral wave period $T_{m-1,0}$.

By combining different structure cross-sections and wave attacks, a total number of 91 simulations was carried out (not all the possible combinations were tested). The main geometrical and hydraulic parameters characterizing the numerical tests are rescaled to prototype units and reported in Table 1.

For each test, 9 numerical wave gauges (wgs) were displaced in the numerical channel to record the free-surface elevation $(h,[\mathrm{~m}])$ and the vertical profiles of the horizontal flow velocity $(u,[\mathrm{~m} / \mathrm{s}])$. The following scheme was setup (see Figure 2):

- $3 \mathrm{wgs}$ were placed in front of the wave generator at a distance of about $19 \mathrm{~m}$ (i.e. at least 1.5 times the highest values of $L_{m-1,0}$ ) for the separation of the incident and reflected wave heights and the calculation of the wave reflection coefficient $\left(K_{r}\right)$ according to the methodology of Zelt and Skjelbreia (1992);

- 2 wgs were set respectively at the off-shore and in-shore edges of the crown wall to reconstruct the vertical profiles of $u$ around the wall;

- the last 4 wgs were displaced inside the reservoir behind the structures to storage the overtopping volumes and calculate the values of the average overtopping discharge $q$ associated to each test.

Specifically, this methodology was followed to calculate $q$. The values of the water depth $h$ accumulated in the reservoir at the end of the simulations and registered at the 4 wgs were multiplied by the width of the reservoir, obtaining 4 estimations of the total overtopped volumes $\left(\mathrm{m}^{3} / \mathrm{m}\right)$. Each volume was then divided by the duration of the simulation itself, deriving thus 4 estimations of $q$ for each test $\left(\mathrm{m}^{3} /(\mathrm{s} \cdot \mathrm{m})\right)$. The final value of $q\left(\mathrm{~m}^{3} /(\mathrm{s} \cdot \mathrm{m})\right)$ is the average of the 4 $q$-values estimated from each $\mathrm{wg}$. This methodology was adopted to get an accurate estimation of $q$ also in case the free-surface elevation was still oscillating inside the reservoir at the end of the simulations, providing different $h$-values at the different wgs. The differences between the values of $q$ obtained from the 4 wgs were always at least 2 orders of magnitude lower than the average values of $q$ themselves, viz the level of uncertainty associated to the average $q$ values can be considered within $5 \%$. These estimations of $q$ are conceptually comparable to the 
measurements of $q$ from the weighing boxes by VD and to the results of the experimental investigations carried at the University of Bologna (Sub-section 2.3).

To further check the reliability of the results of the numerical mode, the values of $q$ derived from the reservoir $\left(q_{\text {res }}\right)$ were compared to the values of $q$ obtained by integrating the flow velocities $(u)$ with the corresponding water depths $(h)$ registered at the 2 wgs placed at the off-shore and in-shore edges of the crown-walls $\left(q_{h u}\right)$. The differences of the results from the 2 methods were at least one order of magnitude lower than the $q$-values themselves, i.e.: $\left|q_{\text {res }}-q_{\text {hu }}\right|<0.1 \cdot q_{\text {res }}\left|q_{\text {res }}-q_{\text {hu }}\right|<0.1 \cdot q_{\text {hu. }}$

The numerical values of $q$ presented hereinafter in this contribution correspond to $q_{r e s}$.

\subsection{The experimental tests}

The experimental part of the new campaign was conducted at the Hydraulic Laboratory of the University of Bologna. The tests were performed in the wave flume (see Figure 3), which is 12 $\mathrm{m}$ long, $0.5 \mathrm{~m}$ wide and $0.7 \mathrm{~m}$ deep. The wave generation is induced by a piston-type wavemaker with a special cuneiform shape, which generates the waves by its vertical movements under the control of the mass conservation law (Galvin, 1964; Wang, 1974). It can be used to generate regular, irregular and focalized waves. The maximum wave height and length which can be generated are respectively of $0.06 \mathrm{~m}$ and $3 \mathrm{~m}$, while the maximum water depth at the wave-maker is $0.4-0.45 \mathrm{~m}$.

The setup of the experiments considered the following works: i) the numerical database collected by Formentin and Zanuttigh (2018a) and ii) the experimental database collected by VD on structures with bns.

Following Formentin and Zanuttigh (2018a), 4 "basic dike" configurations were realized, by combining 2 horizontal berm widths $(B=0.15$ and $0.3 \mathrm{~m})$ and 2 structure slopes $\left(\cot \left(\alpha_{d}\right)=2\right.$ and 4). For each "basic dike", 4 further configurations were investigated, by placing 2 wall heights $\left(h_{w}=0.04\right.$ and $\left.0.05 \mathrm{~m}\right)$ with and without bn $\left(\varepsilon=0\right.$ and $30^{\circ}, \lambda=0$ and 0.375$)$ at the in-shore edge of the berms. By varying the water level $h$ between 0.32 and $0.35 \mathrm{~m}, 2$ berm relative emergences $\left(h_{b} / H_{s}=-0.5\right.$ and 0$)$ were realized for each of the 16 structure configurations. The values of the parameters $\varepsilon, \lambda$ and $h_{w}$ and the ranges of $h_{w} / R_{c}$ were setup following VD and limited by the lab constraints. All the structures and the crown walls were realized in plywood material, which gives a very smooth surface and can be thus characterized by a roughness factor of $\gamma_{f}=1$ (EurOtop 2007). No foreshore slope was set before the dikes in the lab experiments.

Considering the physical constraints of the laboratory and based on the objectives of the research, 2 target wave heights of $H_{s}=0.05$ and $0.06 \mathrm{~m}$ and 2 target wave steepnesses $H_{s} / L_{m-1,0}=0.02$ and 0.03 were tested. The wave attacks were generated with a Jonswap spectrum, by defining the wave height $H_{s}$, the wave period $T_{p}$ and the peak enhancement factor $\gamma=3.3$ in all the tests. Each wave attack was made lasting $480 \mathrm{~s}$ in order to generate a number of waves varying between (at least) 350 and 500 , being the maximum target $T_{p}=1.4 \mathrm{~s}$. 

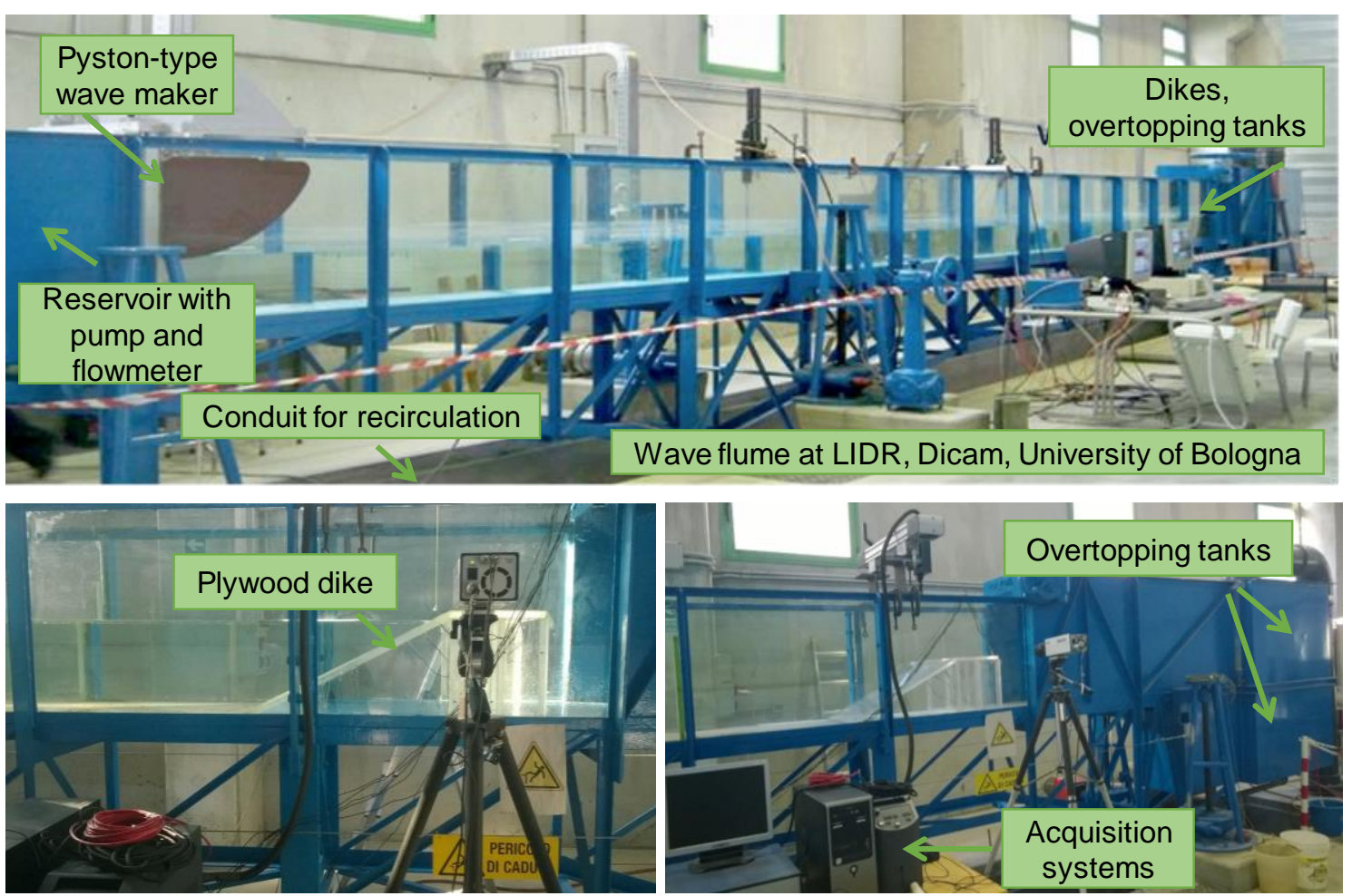

Figure 3 - Wave flume and equipment at the Laboratory of Hydraulics (LIDR), DICAM, University of Bologna.

Overall, 118 tests were performed. The tested conditions is reported in Table 1 in prototype units. Note that a few (10) tests at $h_{b} / H_{s}=-0.5$ and with $H_{s}=0.05 \mathrm{~m}$ were not carried out because the expected overtopping discharge was lower than the minimum measurable due to the lab facility $\left(q<5 \cdot 10^{-5} \mathrm{~m}^{3} /(\mathrm{sm})\right)$.

The lab channel was equipped with:

- 3 resistive wgs placed in the flume at approximately $1.5 \cdot L_{m-10}(\approx 5 \mathrm{~m})$ from the wave-maker to record the free-surface elevations and reconstruct the incident and reflected waves based on Zelt and Skjelbreia (1992); the sampling frequency of $100 \mathrm{~Hz}$ was selected;

- a recirculation system consisting of: a tank for the storage of the overtopping volumes, a conduit and a pump with a flowmeter leading to a precision in the measure of $q$ of approximately $1 \cdot 10^{-5} \mathrm{~m}^{3} / \mathrm{s}$.

The schematic layout of the wave flume, with reference to the position of the structures and of the instruments (wave-maker, wgs, overtopping tank, recirculation conduit, pump for recirculation and flowmeter) is given in Figure 4. For all the structure configurations, the berm off-shore edge was positioned at $10.75 \mathrm{~m}$ from the wave-maker, independently of the berm width and off-shore slope. 


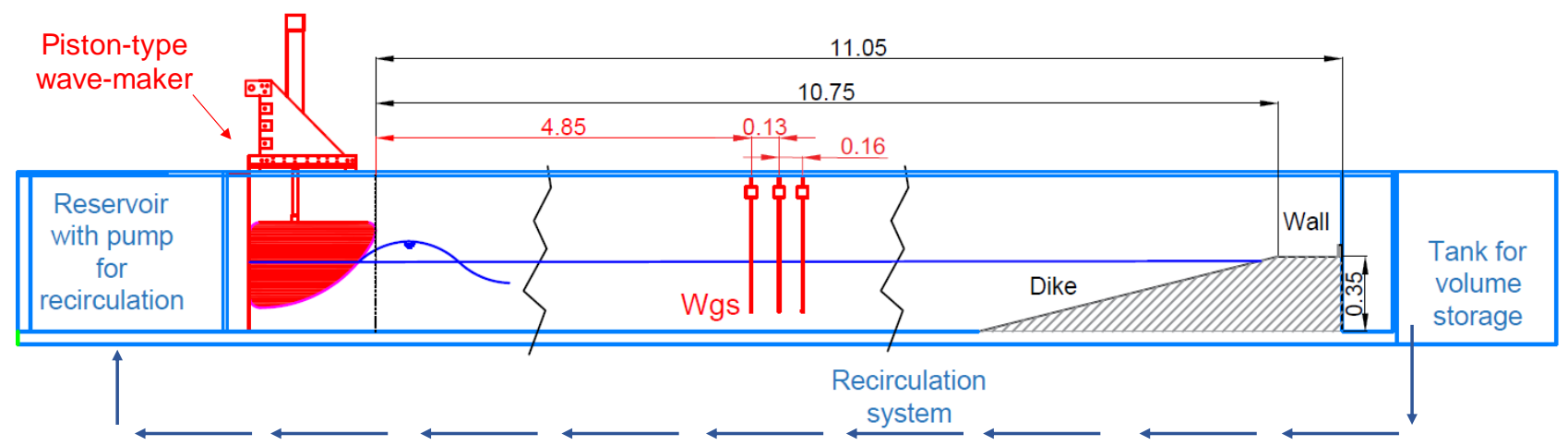

Figure 4 - Schematic layout of the laboratory wave flume and equipment (pump for recirculation, wave gauges and tank for the storage of the overtopping volumes). All the measures are in $\mathrm{m}$.

\subsection{Validation of the numerical model}

The modified version of the code developed to deal with a "dry" landward condition in the numerical channel, was validated by Formentin and Zanuttigh (2018a) against smooth dikes at negative freeboard in both dry and wet conditions, by reproducing existing experimental tests on over-washed and overtopped levees (Hughes and Nadal, 2009) and submerged plywood breakwaters (Cox and Tajziehchi, 2005).

The modified IH2VOF is here validated against smooth berms with crown walls and bullnoses. Objective of the validation is verifying the adequacy of the model to appropriately represent the wave overtopping and reflection processes, giving accurate estimations of $q$ and $K_{r}$. To this purpose, the following experimental tests were reproduced and simulated with the IH2VOF code: i) 4 tests selected from the experiments by VD carried out at the Ghent University ("VD" tests, hereinafter) and ii) 6 of the new experimental tests carried out in the laboratory of Bologna and presented in Sub-section 2.3 (tests "UB", hereinafter). These tests were selected for the validation because representative of the variety of the simulated conditions by considering both the geometrical and the hydraulic features. The main hydraulic and structural parameters characterizing the 10 tests selected for the validation are provided in Table 2 . The 4 VD-tests (1:25 scale) refer to a dike with the same slope $\left(\cot \left(\alpha_{d}\right)=2\right)$ featuring different berm and wall heights $\left(h_{w}=0.06\right.$ and $\left.0.08 \mathrm{~m}\right)$, different freeboards $\left(R_{c}=[0.147 ; 0.233] \mathrm{m}\right)$ and different berm widths $(B=0.33$ and $0.67 \mathrm{~m}$ ). In one of the tests (\#VD-257, see Table 2), the wall includes the bn $\left(\varepsilon=45^{\circ}, \lambda=0.375\right)$. The 6 UB-tests (1:20 scale) consist of 2 different dike slopes $\left(\cot \left(\alpha_{d}\right)=2\right.$ and $4)$, at 2 freeboards $\left(R_{c}=[0.05 ; 0.075] \mathrm{m}\right)$ obtained by varying the berm emergences $\left(h_{b}=0\right.$ and $-0.025 \mathrm{~m})$ and widths $(B=0.15$ and $0.30 \mathrm{~m})$ while keeping the same wall height $\left(h_{w}=0.05 \mathrm{~m}\right)$. No experiment with bn was reproduced from the UB-tests.

For each test belonging to the UB-dataset, Table 2 provides both the target and the measured values of the wave heights $H_{m o}$. This distinction is not made for the wave period because the values of $T_{m-1,0}$ observed in the lab were identical to the target ones. The target values of $H_{m 0}$ and $T_{m-1,0}$ were not available for the VD-dataset. 
As said, the validation focused on the average $q$-values, and not on the distribution of the single overtopping volumes, which were not measured in the lab. For this reason, the numerical simulations of the 10 selected tests were carried out by reproducing the Jonswap spectrums relative to the target wave conditions $H_{m 0}$ and $T_{m-1,0}$ for the UB-tests and to the measured values of $H_{m 0}$ and $T_{m-1,0}$ (the only available) for the UG-tests. Each simulation was made lasting $480 \mathrm{~s}$ and a sample frequency of $20 \mathrm{~Hz}$ was adopted. For the 2 different datasets, 2 numerical channels and 2 mesh grids were set up to exactly and appropriately reproduce the wave flumes and the position of the wgs in the lab. In both cases, the mesh resolution $\Delta z$ was set constant across the numerical channel and equal to $0.005 \mathrm{~m}$, while the horizontal mesh as made variable by keeping the finest grid of $\Delta x=0.005 \mathrm{~m}$ in the area around the structures.

For each test, the resulting $H_{m o}$ and $T_{m-1,0}$ obtained in the numerical channel are reported under the column "Num" in Table 2. By comparing experimental and numerical values of $T_{m-1,0}$, it can be observed that the code generally well reproduces the target wave periods, being in most cases the differences of the order of $0.05 \mathrm{~s}$. There is no systematic tendency to overestimate neither to underestimate $T_{m-1,0}$. As for the wave height, the better agreement among lab and numerical values of $H_{m o}$ is found for the VD-tests, while a certain tendency to underestimation is observed for the UB-tests, especially regarding the tests \#UB-08, \#UB-20 and \#UB-24. Actually, it was already observed that the numerical model tends to reproduce lower wave heights with respect to the target values, especially when the waves are generated in intermediate depth water (see, a.o.: Pilechi et al., 2018; Formentin and Zanuttigh, 2018a), as in the present case. In the case of the UB-tests, the numerical values of $H_{m 0}$ should be compared with the target values of $H_{m 0}$ and not with the $H_{m o}$ measured in the lab.

Table 2 includes the experimental and numerical values of $q$ and $K_{r}$ and the corresponding percentage errors, calculated as relative differences. In case of $q$, the dimensionless quantity $q /\left(g H_{m 0} T_{m-1,0}\right)$ is used to provide a fair comparison between numerical model and experiments, due to the different values of $H_{m 0}$ and $T_{m-1,0}$. The level of accuracy of the numerical model in the representation of the overtopping discharge is assessed by:

- the percentage errors, which are included between $-39 \%$ and $+42 \%$ and between $-55 \%$ and $+26 \%$ for the VD and the UB tests, respectively (see Table 2);

- the values of the determination coefficient $R^{2}$ calculated among measurements and numerical values of $q /\left(g \cdot H_{m 0} \cdot T_{m-1,0}\right)$, which are 0.85 and 0.99 for the datasets VD and UB, respectively.

The agreement among numerical and measured values of $K_{r}$ for the UB tests is represented by:

- the errors, which are included between $-28 \%$ and $+13 \%$ (see Table 2);

- the value of $R^{2}$, which is equal to 0.91 .

The experimental values of $K_{r}$ are not available for the VD-tests.

Overall, the errors associated to both $q$ and $K_{r}$ are randomly distributed (i.e. no systematic overestimation or underestimation is observed) and comparable to the average uncertainty associated to the common predicting methods (see, e.g., Muttray et al., 2006 for $K_{r}$, and EurOtop, 2018 for $q$ ). 
Table 2 - Parameters of the tests selected for the validation of the numerical code. The lab values of $H_{m 0}$ and $T_{m-1,0}$ and the measurements of $q$ and $K_{r}$ (when available) are compared to the corresponding values obtained from the numerical modelling.

\begin{tabular}{|c|c|c|c|c|c|c|c|c|c|c|c|c|c|c|c|c|c|c|c|c|}
\hline \multirow[t]{2}{*}{ Test } & \multirow[t]{2}{*}{ Scale } & \multirow{2}{*}{\begin{tabular}{|c|}
$\begin{array}{c}\mathbf{H}_{\mathrm{m} 0 \text {,target }} \\
\text { [m] }\end{array}$ \\
Target
\end{tabular}} & \multicolumn{2}{|c|}{$\begin{array}{l}\mathbf{H}_{\mathrm{m} 0} \\
{[\mathrm{~m}]}\end{array}$} & \multicolumn{2}{|c|}{$\begin{array}{c}T_{m-1,0} \\
{[s]}\end{array}$} & \multirow[t]{2}{*}{$\begin{array}{c}\cot _{d} \\
{[-]}\end{array}$} & \multirow[t]{2}{*}{$\begin{array}{c}\mathbf{h} \\
{[\mathrm{m}]}\end{array}$} & \multirow[t]{2}{*}{$\begin{array}{c}h_{b} \\
{[\mathrm{~m}]}\end{array}$} & \multirow[t]{2}{*}{$\begin{array}{c}h_{w} \\
{[\mathrm{~m}]}\end{array}$} & \multirow[t]{2}{*}{$\begin{array}{l}R_{\mathbf{c}} \\
{[\mathbf{m}]}\end{array}$} & \multirow[t]{2}{*}{$\begin{array}{c}B \\
{[\mathrm{~m}]}\end{array}$} & \multirow[t]{2}{*}{$\begin{array}{c}\lambda \\
{[-]}\end{array}$} & \multirow[t]{2}{*}{\begin{tabular}{|c|}
$\varepsilon$ \\
{$\left[{ }^{\circ}\right]$}
\end{tabular}} & \multicolumn{3}{|c|}{$\begin{array}{c}\mathbf{q} /\left(\mathrm{gH}_{\mathrm{m0}} \mathrm{T}_{\mathrm{m}-1,0}\right) \\
{[-]}\end{array}$} & \multicolumn{3}{|c|}{$\begin{array}{l}K_{r} \\
{[-]}\end{array}$} \\
\hline & & & $\mathrm{Lab}$ & Num & Lab & Num & & & & & & & & & Lab & Num & $\frac{\text { (Lab-Num) }}{\mathrm{Lab}}$ & Lab & Num & $\frac{\text { (Lab-Num) }}{\text { Lab }}$ \\
\hline \#VD_217 & $1: 25$ & - & 0.158 & .165 & .66 & 1.77 & 2 & 0.42 & -0.173 & 0.06 & 0.233 & 0.67 & 0 & 0 & $8.10 \mathrm{E}-05$ & 7.92E-05 & $-2.2 \%$ & - & 0.693 & - \\
\hline \#VD_232 & $1: 25$ & - & 0.101 & 0.095 & 1.42 & 1.45 & 2 & 0.48 & -0.067 & 0.08 & 0.147 & 0.33 & 0 & 0 & 5.53E-05 & 3.39E-05 & $-38.7 \%$ & - & 0.601 & - \\
\hline \#VD_232 & $1: 25$ & - & 0.159 & 0.157 & 2.05 & 1.89 & 2 & 0.43 & -0.117 & 0.08 & 0.197 & 0.33 & 0 & 0 & 2.45E-04 & 2.61E-04 & $18.6 \%$ & - & 0.651 & - \\
\hline \#VD_257 & $1: 25$ & - & 0.159 & \begin{tabular}{|l|}
0.169 \\
\end{tabular} & 2.05 & 2.12 & 2 & 0.43 & -0.117 & 0.08 & 0.197 & 0.33 & 0.375 & 45 & $1.62 \mathrm{E}-04$ & $2.31 \mathrm{E}-04$ & $42.4 \%$ & - & 0.660 & - \\
\hline \#UB_06 & $1: 20$ & 0.05 & 0.049 & 0.046 & 1.05 & 1.19 & 4 & 0.35 & 0 & 0.05 & 0.050 & 0.3 & 0 & 0 & $3.42 E-05$ & 4.31E-05 & $26.0 \%$ & 0.318 & 0.321 & $1.0 \%$ \\
\hline \#UB_08 & $1: 20$ & 0.05 & 0.065 & 0.049 & 1.27 & 1.19 & 2 & 0.35 & 0 & 0.05 & 0.050 & 0.3 & 0 & 0 & 8.30E-05 & $1.05 \mathrm{E}-04$ & $26.4 \%$ & 0.487 & 0.535 & $9.9 \%$ \\
\hline \#UB_24 & $1: 20$ & 0.05 & 0.069 & 0.051 & 1.05 & 1.10 & 2 & 0.325 & -0.025 & 0.05 & 0.075 & 0.3 & 0 & 0 & 4.41E-05 & 4.99E-05 & $13.0 \%$ & 0.588 & 0.665 & $13.1 \%$ \\
\hline \#UB_04 & $1: 20$ & 0.05 & 0.053 & 0.046 & 1.05 & 1.19 & 2 & 0.35 & 0 & 0.05 & 0.050 & 0.15 & 0 & 0 & $1.16 \mathrm{E}-04$ & $1.41 \mathrm{E}-04$ & $21.8 \%$ & 0.427 & 0.304 & $-28.8 \%$ \\
\hline \#UB_20 & $1: 20$ & 0.05 & 0.059 & \begin{tabular}{|l|}
0.039 \\
\end{tabular} & 1.12 & 1.10 & 2 & 0.325 & -0.025 & 0.05 & 0.075 & 0.15 & 0 & 0 & 4.01E-05 & 3.64E-05 & $-9.2 \%$ & 0.585 & 0.570 & $-2.5 \%$ \\
\hline \#UB_18 & $1: 20$ & 0.05 & 0.052 & \begin{tabular}{|l|}
0.047 \\
\end{tabular} & 1.08 & 1.10 & 4 & 0.325 & -0.025 & 0.05 & 0.075 & 0.15 & 0 & 0 & $1.29 \mathrm{E}-05$ & $5.76 \mathrm{E}-06$ & $-55.4 \%$ & 0.361 & 0.311 & $-14.0 \%$ \\
\hline
\end{tabular}




\section{Application of the existing methods}

This Section proposes the comparison of the new and existing data (i.e. the UB and the VD data) to the methods available from the literature (briefly recalled in Sub-section 3.1), in order to contemporary check the reliability of the data (Sub-section 3.2) and the adequacy of the formulae

5 (Sub-section 3.3).

\subsection{Literature overview}

The literature formulae for the prediction of $q$ at coastal structures have been historically developed separately for breaking and non-breaking waves depending on the values of the breaker parameter $\xi_{m-1,0}$. From now on, following EurOtop (2018), the value of $\xi_{m-1,0}=2$ will be used as transition between breaking and non-breaking.

11 The first wide-ranging applicable method for parametrizing the reduction effects induced by the 12 contemporary presence of one or more structural elements (wall and berm, wall and bn, wall, bn 13 and berm, etc.) on the overtopping at was proposed by VD for non-breaking waves only. This 14 study resulted into a reduction coefficient $y^{*}$ which was meant to be included in the following 15 EurOtop (2007) equation for the prediction of $q$ :

$16 \frac{q_{V D}}{\sqrt{g H_{m 0}^{3}}}=0.2 \cdot \exp \left(-2.3 \cdot \frac{R_{c}}{H_{m 0} \cdot v^{*}}\right), \quad \xi_{m-1,0}>2$

17 The complete formulations for $y^{*}$, which can be synthesized in the following expression,

$18 \quad V^{*}=f\left(\frac{h_{W}}{R_{c}}, \frac{B}{L_{m-1,0}}, \lambda, \varepsilon\right)$

are reported in Appendix 1 of the present contribution (and specifically in the Eqs. A1 to A5). They are valid for smooth structures and non-breaking waves exclusively and each formula has

21 its own range of validity.

22 Van Doorslaer et al. (2016) investigated the possibility to directly apply the original formulations 23 for $y^{*}$ conceived for EurOtop (2007) to the new formula for non-breaking waves presented by 24 Van der Meer and Bruce (2014):

$25 \frac{q}{\sqrt{g H_{m 0}^{3}}}=0.09 \cdot \exp \left(-\left(1.5 \cdot \frac{R_{c}}{H_{m 0} \cdot V_{f} \gamma_{\beta}}\right)^{1.3}\right), \xi_{m-1,0}>2$

where the coefficients $\gamma_{\beta}$ and $\gamma_{f}$ account for, respectively, the wave obliquity and the structure roughness. Based on the promising results by Van Doorslaer et al. (2016), $y^{*}$ was indeed included as it is - i.e. as formulated by VD - in Eq. (2) and adopted by the updated EurOtop

29 (2018) manual:

$\frac{q_{E \text { Eur,2018 }}}{\sqrt{g H_{m 0}^{3}}}=0.09 \cdot \exp \left(-\left(1.5 \cdot \frac{R_{c}}{H_{m 0} \cdot V_{f} v_{\beta} \cdot V^{*}}\right)^{1.3}\right), \quad \xi_{m-1,0}>2, \quad V^{*}=f\left(\frac{h_{W}}{R_{c}}, \frac{B}{L_{m-1,0}}, \lambda, \varepsilon\right)$ 
31 For breaking waves, the EurOtop (2018) approach accounts for the presence of berms and 32 crowns through the 2 coefficients $\gamma_{b}$ and $\gamma_{v}$, respectively, but does not suggest any coefficient 33 for representing the effects of a bn. Following EurOtop (2018), Zanuttigh and Formentin (2018) 34 have recently proposed a new correction factor $y^{* *}$ to extend the applicability of the coefficient $\gamma^{*}$ 35 to breaking waves:

$V^{* *}=\frac{Y^{*}}{\tanh \left(\xi_{m-1,0}\right)}$,

The formulation for $y^{* *}$ has been conceived to make $y^{* *}$ coincide with $y^{*}$ in case of non-breaking waves. Indeed, the denominator $\tanh \left(\xi_{m-1,0}\right)$ goes to 1 when $\xi_{m-1,0}$ goes to $+\infty$, and in the practice when $\xi_{m-10}>2$. The coefficient $\gamma^{\star *}$ from Eq. (5) is meant to work for both breaking and non-breaking waves as follows:

$\frac{q_{\mathrm{ZF}}}{\sqrt{g H_{m 0}^{3}}}=\frac{0.023}{\sqrt{\tan \alpha_{d}}} \cdot \xi_{m-1,0} \cdot \exp \left(-\left(2.7 \cdot \frac{R_{c}}{\xi_{m-1,0} \cdot H_{m 0} \cdot V^{* *}}\right)^{1.3}\right), \quad \xi_{m-1,0} \leq 2, \quad V^{* *}=\frac{V^{*}}{\tanh \left(\xi_{m-1,0}\right)}$

$\frac{q_{Z F}}{\sqrt{g H_{m 0}^{3}}}=0.09 \cdot \exp \left(-\left(1.5 \cdot \frac{R_{c}}{H_{m 0} \cdot V^{* *}}\right)^{1.3}\right), \xi_{m-1,0}>2, \quad V^{* *}=\frac{\gamma^{*}}{\tanh \left(\xi_{m-1,0}\right)}$

In summary:

- the original method proposed by VD consists of the application of Eq. (1), by including $V^{*}$ as provided by Eqs (A1-A5); this method will be referred as "VD method" and the corresponding predictions as $q_{v D}$;

- the method proposed by EurOtop (2018) consists of the application of Eq. (4) including $\gamma^{*}$ as formulated by VD; this will be the "Eur2018 method" and the corresponding predictions as named $q_{\text {Eur,2018; }}$;

- the method proposed by Zanuttigh and Formentin (2018) consists of Eqs. (6a) and (6b), and essentially coincides with the EurOtop (2018) method in case of non-breaking waves; this method will be named "ZF method" and the related predictions $q_{Z F}$.

\subsection{Characterization of the new data}

The UB-data presented in Section 2 are here checked against the literature methods VD, Eur 2018 and ZF. The analyses are organized as follows:

- the non-breaking tests are compared to the curves representing Eq. (1) and Eq. (6b), in Figure 5 and 6b, respectively;

- the breaking tests are compared in Figure $6 \mathrm{a}$ to the curve representing Eq. (6a);

- the graphical comparison with Eq. (4) is not provided as it is equivalent to Eq. (6b);

- in each chart of Figures 5 and 6 , the data are further distinguished between tests on structures with berms and walls (void squares) and tests on structures with berms, walls and bns (filled-in circles); 
- for each group of tests, the agreement among the measured values of $q$ and the corresponding predictions obtained with the 3 methods is quantitatively assessed by means of the coefficient of determination $\left(R^{2}\right)$, whose values are reported in Table 3 (upper part). The indexes associated to the methods VD (Eq. 1) and Eur 2018 (Eq. 4) are given for the data on non-breaking waves only, because all the other datasets include tests with breaking waves which cannot be represented by Eqs. (1) and (4). The $R^{2}$ performance index was preferred to the root mean squared error ( $r m s e)$ as $R^{2}$ is dimensionless and therefore independent of the magnitude of the $q$-values, which vary of several order of magnitudes (from $10^{-7}$ to $10^{-2} \mathrm{~m}^{3} /(\mathrm{sm})$ ) across the datasets. $\mathrm{R}^{2}$ allows therefore a meaningful comparison among different datasets and predicting methods.

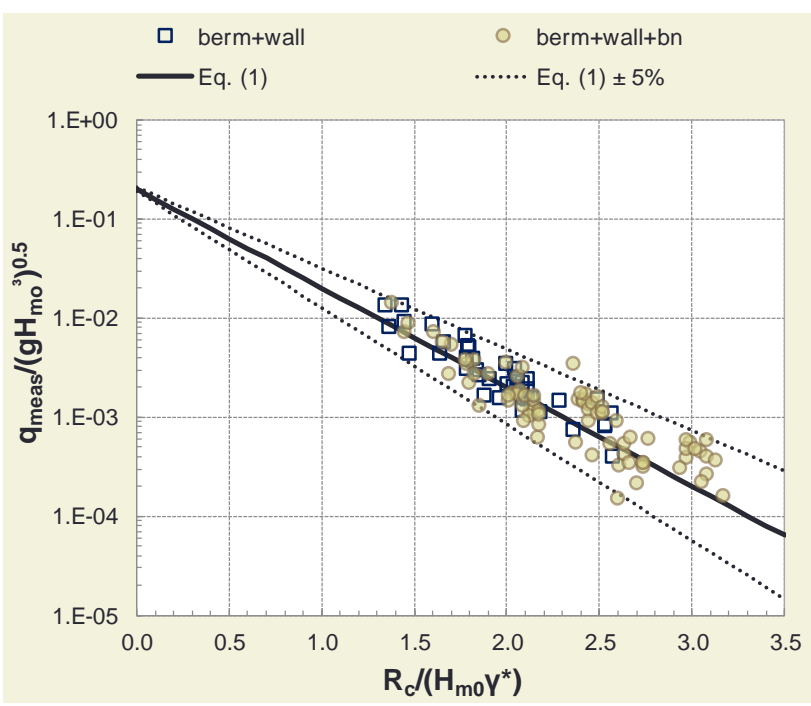

Figure 5 - Dimensionless discharges $q_{\text {meas }} /\left(\mathrm{gH}^{3}{ }_{\mathrm{mo}}\right)^{0.5}$ from UB-data as functions of $R_{d} H_{m 0}$ compared to the curve (continuous line) representing the VD method, Eq (1). The dotted lines are the $90 \%$ confidence bands.
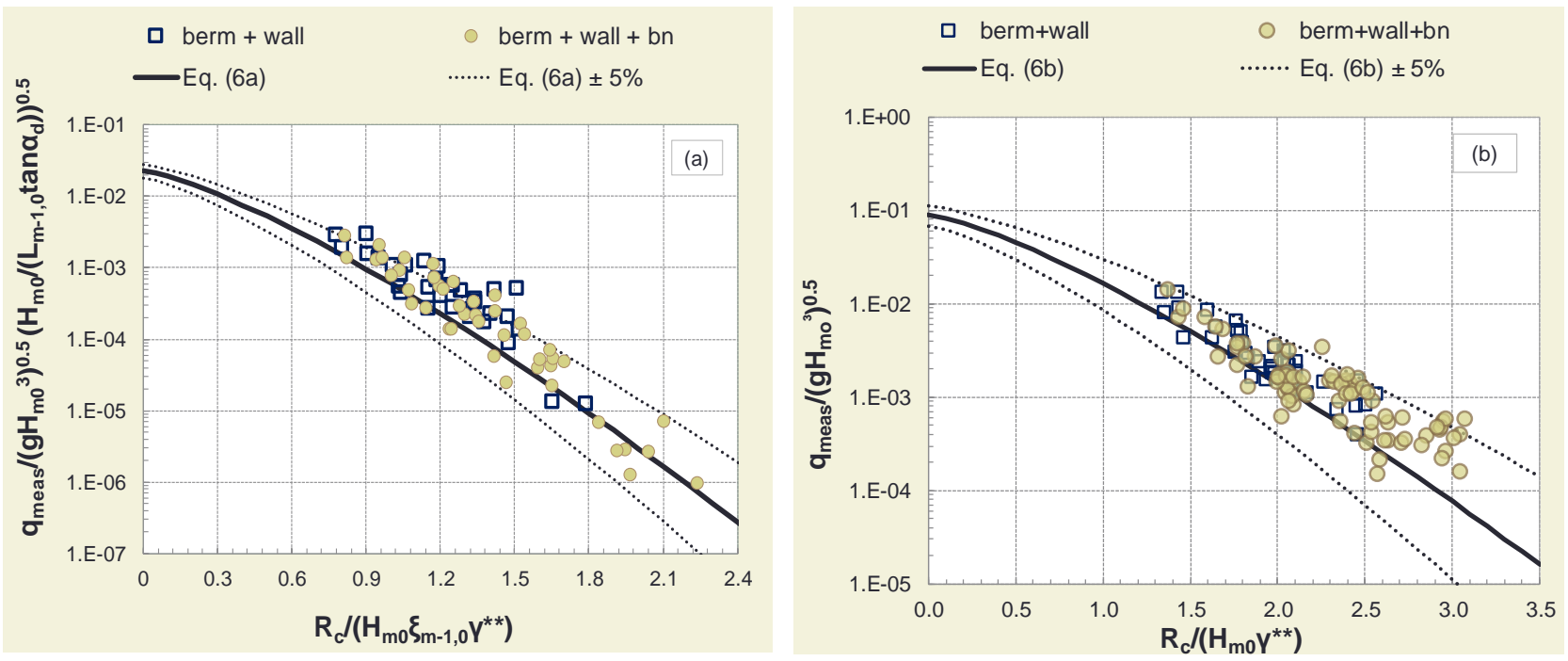

Figure 6 - Dimensionless discharges $q_{\text {meas }} /\left(\mathrm{gH}^{3}{ }_{\mathrm{mo}}\right)^{0.5}$ from UB-data as functions of $R_{d} H_{m 0}$ compared to the curve (continuous line) representing the ZF method in case of breaking (panel a, Eq. 6a) and non-breaking (panel b, Eq. 6b) conditions. The dotted lines are the $90 \%$ confidence bands. 

applied to the prediction of $q$ for the datasets involved in the GP training. Error indices $R^{2}$ (coefficient of determination) and SK (skewness).

\begin{tabular}{|c|c|c|c|c|c|c|c|c|}
\hline \multirow{2}{*}{$\begin{array}{c}\begin{array}{c}\text { Dataset } \\
\text { (\#nr. of tests) }\end{array} \\
\text { UB database }\end{array}$} & \multicolumn{4}{|c|}{$R^{2}$} & \multicolumn{4}{|c|}{ SK } \\
\hline & $\begin{array}{c}q_{G P} \\
(\text { Eq. 12) }\end{array}$ & $\begin{array}{c}q V D \\
(\mathrm{Eq} .1)\end{array}$ & $\begin{array}{l}q_{\text {Eur,2018 }} \\
\text { (Eq. 4) }\end{array}$ & $\begin{array}{c}q Z F \\
\text { (Eq. 6) }\end{array}$ & $\begin{array}{c}q_{G P} \\
(\text { Eq. 12) }\end{array}$ & $\begin{array}{c}q V D \\
(\text { Eq. 1) }\end{array}$ & $\begin{array}{l}q_{\text {Eur,2018 }} \\
\text { (Eq. 4) }\end{array}$ & $\begin{array}{c}q z F \\
\text { (Eq. 6) }\end{array}$ \\
\hline All data (209) & 0.831 & - & - & 0.769 & 0.73 & & - & -1.91 \\
\hline Experimental (118) & 0.908 & - & - & 0.916 & 0.60 & & - & -1.70 \\
\hline Numerical (91) & 0.760 & - & - & 0.675 & 1.05 & & - & -0.91 \\
\hline Breaking (82) & 0.922 & - & - & 0.880 & 1.50 & & & -1.76 \\
\hline Non-breaking (127) & 0.764 & 0.732 & 0.722 & 0.682 & 0.46 & -0.53 & -1.89 & -1.44 \\
\hline Berm+wall (75) & 0.906 & - & - & 0.872 & 1.03 & & - & -1.53 \\
\hline Berm+wall+bn (134) & 0.779 & - & - & 0.675 & -0.26 & & - & -1.95 \\
\hline VD Database & $\begin{array}{c}q_{G P} \\
\text { (Eq. 12) }\end{array}$ & $\begin{array}{c}q V D \\
(\text { Eq. 1) }\end{array}$ & $\begin{array}{l}q_{\text {Eur,2018 }} \\
\text { (Eq. 4) }\end{array}$ & $\begin{array}{c}q Z F \\
(\text { Eq. 6) }\end{array}$ & $\begin{array}{c}q_{G P} \\
\text { (Eq. 12) }\end{array}$ & $\begin{array}{c}q V D \\
(\text { Eq. 1) }\end{array}$ & $\begin{array}{l}q_{\text {Eur,2018 }} \\
\text { (Eq. 4) }\end{array}$ & $\begin{array}{c}q_{Z F} \\
\text { (Eq. 6) }\end{array}$ \\
\hline All data (596) & 0.948 & 0.960 & 0.956 & 0.956 & 1.30 & 0.92 & -1.56 & -1.76 \\
\hline Wall (119) & 0.969 & 0.974 & 0.973 & 0.974 & 1.42 & 1.68 & -1.59 & -2.01 \\
\hline Wall+bn (176) & 0.967 & 0.979 & 0.973 & 0.979 & 1.93 & -2.03 & -2.24 & -2.21 \\
\hline Berm (63) & 0.988 & 0.993 & 0.992 & 0.993 & -1.12 & 2.38 & -1.66 & -1.77 \\
\hline Berm+wall (137) & 0.960 & 0.969 & 0.968 & 0.980 & 2.25 & 2.18 & -0.97 & -1.25 \\
\hline Berm+wall+bn (101) & 0.851 & 0.880 & 0.871 & 0.868 & -0.15 & 0.78 & -1.10 & -1.38 \\
\hline
\end{tabular}

Figure 5 shows that the VD method provides a very good representation of the UB-data, as almost all the tests are included within the $90 \%$ confidence bands (dotted lines) and are symmetrically distributed around the fitting curve. No evident heteroscedasticity is found towards any of the data groups, both considering the numerical and the experimental tests. In the 2 charts of Figure 6 the data tend instead to be distributed above the fitting curves, exceeding in some cases the upper $90 \%$ confidence bands. This general trend suggests that the ZF method - and the Eur 2018 method - underestimate, on average, the values of $q$, leading to a non-conservative approach. This shortcoming cannot be appreciated from the $\mathrm{R}^{2}$ values of Table 3 , as this index is suitable to assess the accuracy of the predictions but cannot account for the symmetry of their distribution. Indeed, all the 3 predicting methods are associated with very similar and relatively high values of $R^{2}(0.732,0.722$ and 0.682 for VD, Eur2018 and ZF, respectively, applied to the non-breaking dataset), in line with the modest scatter of the points in the Figures 5 and 6 .

\section{8}

To assess the symmetry of the error distribution, i.e. of the symmetry of the distribution of the differences between predictions and measurements of $q$, the Pearson's coefficient of skewness $(\mathrm{SK})$ is adopted:

$$
\mathrm{SK}=\frac{\mathrm{n}}{(\mathrm{n}-1)(\mathrm{n}-2)} \cdot \sum_{\mathrm{j}=1}^{\mathrm{n}}\left[\left(\frac{x_{j}-\mu}{\sigma}\right)^{3}\right]
$$

where: $x_{j}=q_{\text {pred }, j}-q_{\text {meas }, j}$ is the difference (or error) between the predicted and the measured value of $q$ associated to the $j$-th test; $\mathrm{n}$ is the number of tests; $\mu$ and $\sigma$ are, respectively, the mean and the standard deviation of the distribution of the error values. In case of a perfectly symmetric distribution, $\mathrm{SK}=0$, while in case of distributions biased towards positive $\left(x_{j}=q_{\text {pred } j-}-q_{\text {meas }, j}>0\right)$ and negative $\left(x_{j}=q_{\text {pred }, j}-q_{\text {meas }, j}<0\right)$ errors, $S K$ is respectively greater and lower than 0 . Therefore, a positive value of SK indicates that the predicting method is affected by an overestimation bias, 
while a negative value of SK indicates an underestimation bias. The absolute value of SK indicates the "degree" of asymmetry: the greater $|\mathrm{SK}|$, the more pronounced the bias.

The values of SK are provided (when possible) for each group of data in Table 3 next to the corresponding values of $R^{2}$. All the values of $S K$ associated to $q_{\text {Eur2018 }}$ and $q_{Z F}$ are negative and relatively high in terms of absolute values $(-1.95 \leq S K \leq-0.91)$, confirming that both the predicting methods, on average, underestimate the values of $q$ in case of composite structures. As for $q_{V D}$, the value of $S K=-0.53$ obtained for the non-breaking dataset reveals that Eq. (1) gives significantly smaller underestimations of $q$, as it is graphically evident from Figure 5.

\subsection{Limits of the existing predicting methods}

To further investigate the adequacy of the methods Eur 2018 and ZF, Eqs. (4) and (6) were also applied to the prediction of the existing data by VD, i.e. the same data which were used to develop the formulations Eqs. (4) and (6) themselves. The values of $R^{2}$ and SK associated to the predictions of the data composing the whole database by VD and of the 5 subsets of tests grouped by structure types (dikes with walls, dikes with walls and bns, dikes with berms, dikes with berms and walls and dikes with berms, walls and bullnoses) are reported in the lower part of Table 3. Table 3 includes also the values of $R^{2}$ and SK associated to the original VD method (Eq. 1), which were calculated to provide a comparison with the other formulae.

The values of $R^{2}$ obtained for the application to the VD database, ranging between 0.868 and 0.993, are particularly high if compared to the values obtained for the UB-database and all the methods seem to be characterized by very similar accuracy. A relevant difference occurs between the values of SK: whereas all but one the values of SK associated to $q_{V D}$ are positive $\left(-1.90 \leq S K \leq 2.38\right.$, with $S K=0.92$ for the whole database), all the values of $S K$ associated to $q_{z F}$ or $q_{\text {Eur2018 }}$ are again systematically negative $(-2.24 \leq \mathrm{SK} \leq-0.97$, with $\mathrm{SK}=-1.56$ and $\mathrm{SK}=-1.76$ on the whole database, respectively).

To better illustrate the underestimation shortcoming associated to $q_{E u r 2018}$ and $q_{z F}$, the predictions of $q$ obtained with the 2 methods are directly compared to the corresponding measured values in Figure 7. Figure 7a presents the distribution $q_{E \text { Eur,2018 }} v s q_{\text {meas }}$ relative to the VD data, while Figure $7 \mathrm{~b}$ shows the distribution $q_{z F} v s q_{\text {meas }}$ relative to the new experimental and numerical data. Both the plots show a clear underestimation of the $q_{\text {meas }}$-values, in line with the values of SK. In some cases, $q_{E u r 2018}$ and $q_{Z F}$ can be even one order of magnitude lower than the corresponding $q_{\text {meas. }}$ In Figure $7 \mathrm{a}$, the points are "rotated" around the bisector line, revealing that the underestimations of by $q_{E u r 2018}$ tend to decrease with increasing $q_{\text {meas }}$ values. The data in Figure $7 \mathrm{~b}$ are instead "shifted" below the bisector line, suggesting that the underestimations of $q_{z F}$ are on average of the same order of magnitude, independently of the values of $q_{\text {meas }}$. 

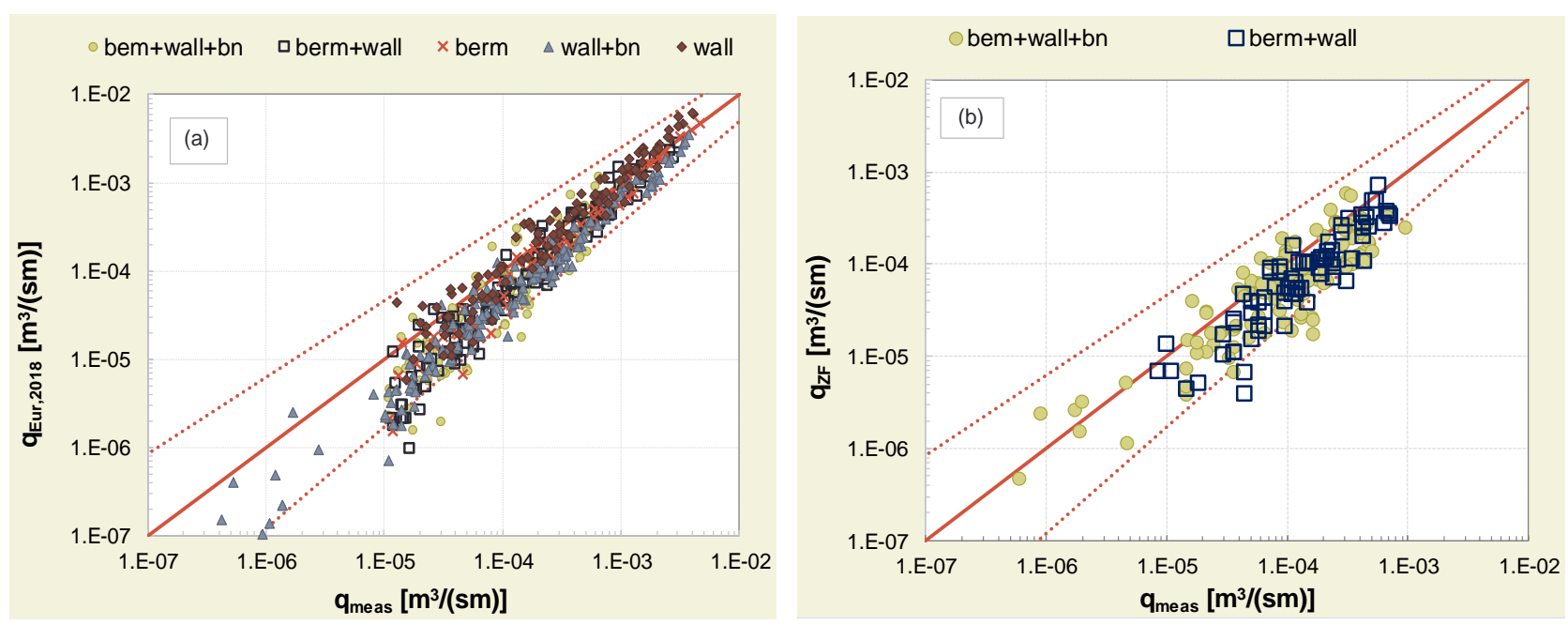

144 Figure 7 - Measured values of $q\left(q_{\text {meas }}\right)$ belonging to the datasets VD (panel a) and UB (panel b) compared to the corresponding predictions obtained with the methods $\operatorname{Eur}_{2018}$ (panel a) and ZF (panel b). The continuous bisector lines represent the optimal agreement among predictions and measurements, while the dotted lines are the $90 \%$ confidence bands.

In synthesis, the combined analyses of Figures 5, 6 and 7 and of Table 3 indicate that:

- the original coefficient $\gamma^{*}$ proposed by VD and optimized for EurOtop (2007) gives an adequate and conservative representation of the new UB-data, though it is limited to the non-breaking wave conditions only;

- the coefficient $Y^{*}$, and its extension to breaking waves proposed by ZF through the coefficient $Y^{* *}$, may require an update or a re-calibration of the numerical coefficients to be successfully applied to EurOtop (2018). These formulae indeed significantly underestimate both the UB and the VD data.

A different approach is therefore necessary for representing the reductive effects of crown walls and bns, which should be:

- conceived and fitted to be applicable to the most recent version of the EurOtop (2018) equations, provide conservative estimations of $q$;

- directly applicable to both the formulae for breaking and non-breaking waves;

- of possibly easier application than the method by VD (see Appendix 1).

\section{The new formula based on Genetic Programming}

A new formula for the estimation of the correction factor $y$ accounting for the reductive effects of the crown walls and bullnoses to be included in the EurOtop (2018) formulae for the prediction of $q$ in case of both breaking and non-breaking waves was developed and it is presented in this Section. The formula is the result of physically-based optimization of an existing Genetic Programming algorithm (Searson, 2009) to the new numerical and experimental data collected by the authors and to the existing data by VD. The raw model obtained with the GP has been slightly modified, based on the available data and information form the literature, to better 
represent the wave breaking phenomenon and achieve a simpler and more operative 173 formulation.

174 The final formula is proposed and described in Sub-section 4.4, after a brief description of the 175 key elements of GP (Sub-section 4.1), the characterization of the data used for the GP training 176 and the illustration of the GP scheme and parameters adopted (Sub-section 4.2). Sub-section 1774.3 discusses limits and advantages of GP.

\subsection{Elements of GP}

179

The Genetic Programming (GP) is a biologically-inspired computational method belonging to the family of the "machine learning" tools. Similarly to other Artificial Intelligence methods, it is an advanced regression model that creates and trains computer programs to perform a task by learning the information from empirical records. The task of the GP is to build an empirical mathematical formulation that can be used to model a specific physical process or predict a specific parameter. For this reason, it can be said that the GP performs a "symbolic regression" analysis of the data. In the case of the present contribution, the task is building a formula for the estimation of the correction factor $y^{*}$ to be included in the Eqs. (1) and (2). The difference from the traditional regression analysis is that the symbolic analysis automatically creates and develops the parameters and the form of the model to be built. This is automatically done by the GP by supplying:

- a sufficient number of observation data to perform the regression on, which will represent the "training" and "testing" data;

- the potential parameters (or variables) which the coefficient $y$ is supposed to depend on;

- a number of analytical functions that may fit the problem (e.g. square root, power, exponential, hyperbolic tangent, etc.);

- the main parameters and features required for the setup the GP algorithm.

The GP algorithm starts by randomly generating a "first population" of several formulae (or "individuals") containing the variables (or "genes") defined by the user. Each formula includes a random selection of the variables, linked each other through attempt functions selected from the ones initially defined by the user. The number and the form of the formulae composing the first population are then iteratively mutated, "generation by generation", until the "best performing formula", i.e. the formula that solves the task best, is found. At each generation, the adequacy of each formula of the current population to fit the task is checked against the data selected for the training and the testing, and a next generation is created. The creation of a new generation is inspired to the biological process of natural evolution, where the "parental" genetic information is passed down through generations by the following three key processes: reproduction, mutation and cross-over.

A very clear and effective description of the conceptual steps composing a GP algorithm is given by Gaur and Deo (2008), while a detailed explanation of the various concepts related to GP can be found in Koza (1992). A brief synthesis of the main terms and concepts characterizing the GP is reported in Appendix 2 with reference to the scheme of Figure 8. 
The result of the application of GP is a regression model or formula consisting of several terms (genes) added up together. Each gene is a function of one or more parameters governing the problem (e.g., $\tanh \left(\xi_{m-1,0}\right)$, or $\left.\exp \left(-R_{d} / H_{m 0^{1}} 1 / \xi_{m-1,0}\right)\right)$ multiplied by a weight factor. In the GP language, such formula is a "multigene individual", where the output variable (e.g., the coefficient $v)$ is predicted by the weighted output of each of the genes plus a bias term. Mathematically, this can be written as:

$y=x_{0}+w_{1} \cdot x_{1}+w_{2} \cdot x_{2}+\ldots+w_{n} \cdot x_{n}$,

where $x_{0}$ is the bias and $w_{1}, w_{2} \ldots, w_{n}$ are the gene weights and $n$ is the number of genes $x_{1}, x_{2}$, $\ldots, x_{n}$. The weights are regression coefficients automatically determined by a least squares procedure for each multigene individual.

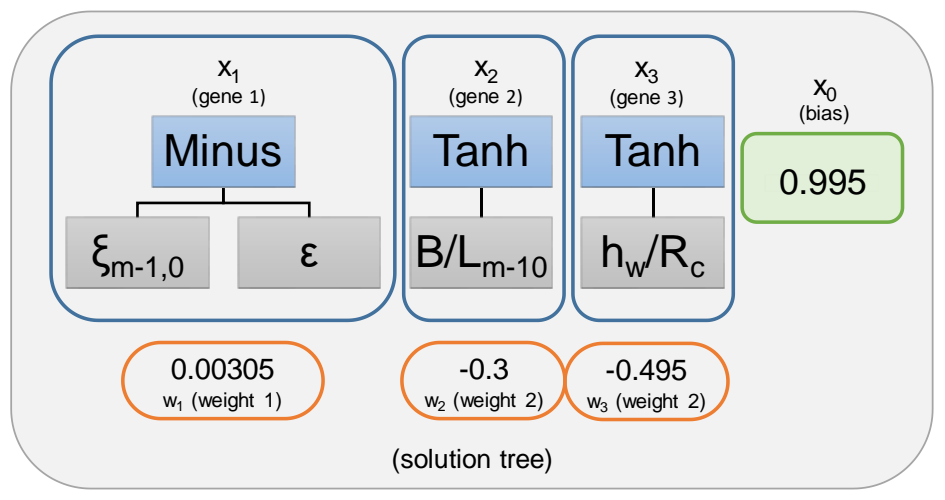

Figure 8 - Example of a GP solution formula $\left(0.003\left(\xi_{m-1,0-\varepsilon}\right)-0.300 \tanh \left(B / L_{m-1,0}\right)-0.495\right.$ $\left.\tanh \left(h_{w} / R_{c}\right)+0.995\right)$ symbolically represented as a tree. The solution includes 3 genes and the bias.

\subsection{GP scheme, parameters and training dataset}

The GP technique was applied in this contribution for the specific task of building an analytical formulation for the estimation of the factor $y^{*}$ to be included in Eqs. (1) and (2). The setup of the GP started with the identification of the data to train and test the computer program. To this purpose, the following databases were considered:

- the experimental database by VD (596 tests);

- the new numerical and experimental databases presented in this contribution (UB tests), which were specifically col

Other databases on overtopping at crown walls and bns available from the literature were not considered for the GP training because consisting of different structure cases (e.g., tests on seawalls, seawalls with parapet and double seawalls by Oumeraci et al., 2004) or because were specifically left apart to check the generalization of the formula (e.g., the tests on recurved return walls by Owen and Steele, 1991), provided in Sub-section 5.3. Only smooth structures were used because the amount of the available data on rubble mound structures with crown walls and bns was exiguous (ratio $\approx 1: 10$ with respect to the number of smooth structures) and insufficient to properly train the GP. 
A sketch of the variety of the structure types considered for the GP training is illustrated in Figure 9 , while the ranges of the hydraulic and geometrical parameters of the tested conditions are reported in Table 2 for the 3 databases.
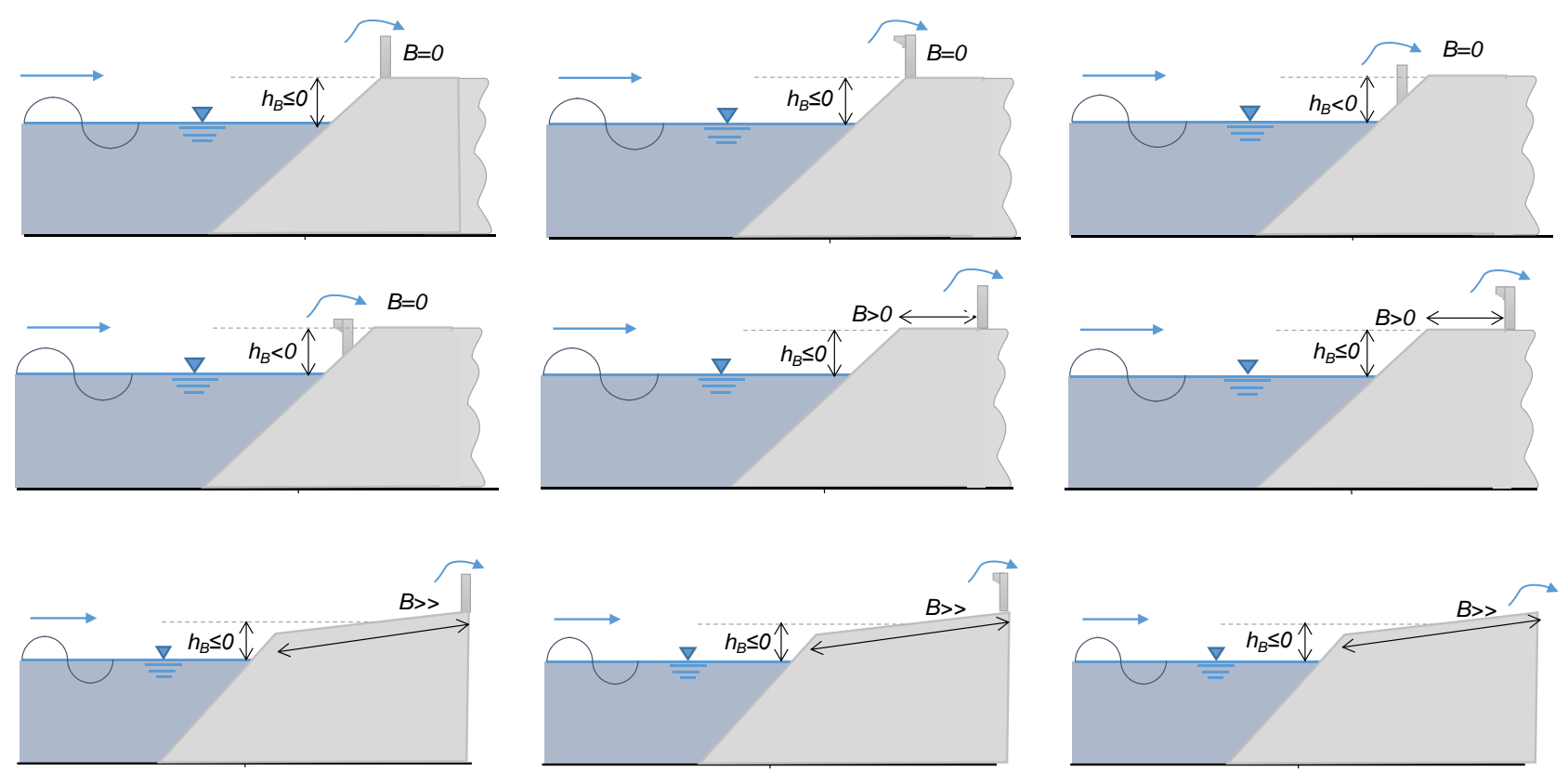

Figure 9 - Schemes of the different structure cross-sections involved in the GP training.

The target parameters for the application of GP are the values of the $V^{*}$ factor to be predicted through a new formulation. For each of the training tests, it was therefore necessary to "build" a corresponding target value of $\gamma^{*}$. This was calculated from the experimental values of $q$ and the other parameters $R_{c}, H_{m 0}$, $\tan \left(\alpha_{d}\right), \xi_{m-1,0}$ available for each test of the training dataset, by making $V^{*}$ explicit from EurOtop (2018), i.e. by means of the following expressions:

$V^{*}=-\left[\ln \left(\frac{q}{\sqrt{g H_{m 0}^{3}}} \cdot \frac{\sqrt{\tan \left(\alpha_{d}\right)}}{0.023 \cdot \xi_{m-1,0}}\right)\right]^{-\frac{1}{1.3}} \cdot \frac{2.7 \cdot R_{c}}{H_{m 0} \cdot \xi_{m-1,0}}$, if $\xi_{m-1,0} \leq 2$

$V^{*}=-\left[\ln \left(\frac{q}{\sqrt{g H_{m 0}^{3}}} \cdot \frac{1}{0.09}\right)\right]^{-\frac{1}{1.3}} \cdot \frac{1.5 \cdot R_{c}}{H_{m 0}}$, if $\xi_{m-1,0}>2$

In other terms, the values of $\gamma^{*}$ obtained from Eq. (9a-9b) are the values of $\gamma^{*}$ that make the predictions of $q$ by EurOtop (2018) coincide to the corresponding experimental values. The reduction coefficients $\gamma_{f}, \gamma_{b}$ and $\gamma_{\beta}$ do not appear in Eq. (9) as their values equal 1 for all the tests.

The second step of the setup phase consisted of the individuation of the potential input parameters that may influence the values of the output parameter $V^{*}$. The selection of the parameters started from the analysis of the state-of-art formulae by VD, and evolved in a "trial and error" process driven by the results of an in-depth sensitivity analysis to the effectiveness of a number of different combinations of parameters. Specifically, the following parameters were 
initially selected: $h_{b} / H_{m o}, B / L_{m-10}, R_{d} / H_{m 0}, h_{w} / R_{c}, \varepsilon, \lambda, \cot \left(\alpha_{d}\right), \cot \left(\alpha_{i n c l}\right), s_{m-1,0}, \xi_{m-1,0}$. With respect to the parameters adopted by VD, the structure slopes $\cot \left(\alpha_{d}\right), \cot \left(\alpha_{\text {incl }}\right)$ and the breaker parameter $\xi_{m-1,0}$ were added to account for the breaking wave conditions included in the UBdataset. The sensitivity analysis was carried out by training the GP several times on the same dataset and by using each time a different set of these input parameters. The GP automatically evolves in a program that assigns the proper weight to each parameter, measuring their effective influence on the output. If a parameter is not influencing at all, it is even not included in the final formulation (i.e., its weight is 0 ). The outcomes of the sensitivity analysis suggested that the best performing formulations for predicting $\gamma^{*}$ include (only) the following parameters: $B / L_{m-10}, h_{w} / R_{c}$, $\varepsilon, \xi_{m-1,0 .}$ In other words:

$\gamma^{*}=\gamma^{*}\left(B / L_{m-10}, h_{w} / R_{c}, \varepsilon, \xi_{m-1,0}\right)$.

In this expression, it is evident that the parameter $\xi_{m-1,0}$ outperforms the single parameters $s_{m-1,0}$ and $\cot \left(\alpha_{d}\right)$ as they are embedded in its definition, while the combination of $\varepsilon$ and $B / L_{m-10}$ replaces $\cot \left(\alpha_{i n c l}\right)$. An interesting result is that the parameters $h_{b} / H_{m 0}, \lambda$ and $R_{d} / H_{m o}$ are apparently not (or negligibly) affecting $\gamma^{*}$.

The third step regarded the definition of the functions that might compose Eq. (10). Also in this case, the same trial and error technique was applied. The most common functions (times; minus; plus; divide; square; cube; exp; power; square root; tanh; In) were tested, and, based on the formulations obtained during the sensitivity analysis, a few were discarded (root, power, In). A sensitivity analysis was then performed to select also the most suitable fitness function among rmse, $\mathrm{R}^{2}$ and SK. rmse was discarded because affected by the order of magnitude of the $q$ values, giving thus less "importance" to the lower values of $q$, while SK focuses exclusively on the symmetry of the error distribution disregarding its accuracy. For this reason, $\mathrm{R}^{2}$ was chosen.

The final step consisted of the configuration of the proper GP algorithm and the development of the code. To this purpose, the GP package tool "GPTIPS" developed by Searson (2009) for use with Matlab was adopted. This tool, which is freely downloadable on http://gptips.sourceforge. net and easily installable in a standard pc, essentially consists of a library of Matlab functions that can be customized to build an own model of GP. GPTIPS provides also a number of convenient functions for exploring the population of evolved models, investigating model behaviour, postrun model simplification and export to different formats (e.g. graphics file, LaTex expression, symbolic math object or standalone $\mathrm{m}$ file). The use of GPTIPS, which only requires a basic knowledge of Matlab environment and programming language, is eased by a detailed User Guide, which explains how to customize the code and setup the parameters of interest.

The features selected in the final configuration of the GP are reported in Table 4. Some of the terms included in this Table are derived from the language of GPTIPS. An explanation to the terms is given in Table 4 itself, while reference to the GP functions and working principle is provided in Sub-section 4.1.

The results of the application of GP do not consist of a unique solution (unique individual), but in a set of solutions (population of individuals of the last generation) that fit the task with different degrees of accuracy and complexity. More precisely, the GP provides the number of solutions 
that fits the final population size (in the present case 500, see Table 4). GPTIPS selects the "best performing" individuals among the overall population of the last generation, based on a criterion of maximum accuracy and minimum complexity. This means that the "best performing" individuals are not necessarily the ones which optimize the fitness function, but the ones which reach a good fitness score without exceeding in complexity. The level of complexity of an individual is determined by the number of genes involved, in the number and types of functions included and in the "tree depth", i.e. the number of non-linear genes and the level of non-linearity. A too high level of complexity would seriously affect the solution generalization.

Table 4 - Parameters and relative values adopted in the final setup of the GP. Part of the terminology is specific of the GPTIPS tool (Searson, 2009).

\begin{tabular}{|c|c|c|}
\hline Run parameter & Description of the parameter & Value \\
\hline $\begin{array}{l}\text { Elapsed time } \\
\text { [hh:mm:ss] }\end{array}$ & $\begin{array}{c}\text { Time required to run the GP and find the "best performing } \\
\text { individual" or to break the algorithm based on the stopping } \\
\text { criteria }\end{array}$ & 00:01:02 \\
\hline Run timeout [s] & $\begin{array}{l}\text { Maximum time allowed for creating a new generation that } \\
\text { performs better than the previous one }\end{array}$ & 30 \\
\hline Population size & $\begin{array}{l}\text { Number of individuals composing the population at the first } \\
\text { generation }\end{array}$ & 500 \\
\hline $\begin{array}{l}\text { Crossover } \\
\text { probability }\end{array}$ & $\begin{array}{l}\text { Probability of creating a next-generation individual } \\
\text { exclusively from the cross-over of the genes of two } \\
\text { parental individuals }\end{array}$ & 0.84 \\
\hline $\begin{array}{c}\text { Mutation } \\
\text { probabilities }\end{array}$ & Probability of getting a gene mutation at each generation & 0.14 \\
\hline Elite fraction & $\begin{array}{c}\text { Fraction of individuals belonging to a population that are } \\
\text { directly passed to the next generation (without cross-over } \\
\text { and mutation) }\end{array}$ & 0.3 \\
\hline Function set & $\begin{array}{l}\text { Functions initially defined by the user that may compose } \\
\text { the predicting model }\end{array}$ & $\begin{array}{l}\text { times; minus; plus; divide; } \\
\text { square; cube; exp; power; square } \\
\text { root; tanh; }\end{array}$ \\
\hline Max. generations & $\begin{array}{l}\text { Maximum number of generations } \\
\text { allowed before breaking the GP algorithm }\end{array}$ & 300 \\
\hline $\begin{array}{l}\text { Generations } \\
\text { elapsed }\end{array}$ & $\begin{array}{c}\text { Total number of generations created before finding the } \\
\text { "best performing individual" or satisfying the stopping } \\
\text { criteria }\end{array}$ & 88 \\
\hline Input variables & Physical parameters initially supplied by the user & 5: $B / L_{m-10} ; h_{w} / R_{c} ; \varepsilon ; \xi_{m-1,0} ; R_{c} / H_{m 0}$ \\
\hline Max. genes & $\begin{array}{l}\text { Maximum number of genes that each individual may } \\
\text { contain }\end{array}$ & 5 \\
\hline Max. tree depth & Parameter used to limit the size of the initialized trees & 2 \\
\hline
\end{tabular}

Table 5 reports the 4 best performing solutions for the formulation of $\gamma^{*}$ individuated with GP. In this Table, each solution is assigned an ID, a fitness score (the coefficient of determination $\mathrm{R}^{2}$ ) and a complexity score. The level of complexity of all the solutions in Table 5 is relatively low as it was limited by setting the maximum tree depth equal to 2 and the maximum number of genes equal to 5 . At the same time, the fitness scores obtained on the testing data are relatively high. The solution $\# 504$ presents the best fitness score $\left(R^{2}=0.852\right)$ but is the most complicated (complexity 18), as it involves non-linear genes $\left(0.479 \cdot B / L_{m-1,0} \xi_{m-1,0}\right.$ and $\left.0.044 \xi_{m-1,0} h_{w} / R_{c}\right)$ and a higher number of genes with respect to the solutions \#272 and \#280. Overall, solution \#272 represents the best compromise between fitness score and complexity (11), as it does not 
involve non-linear genes but its $R^{2}$ value $(0.834)$ is comparable to highest fitness score of solution \#504. To individuate the best solution an "overall score" was assigned to each solution. The overall score - whose values are reported in Tab. 5 - was calculated as the quotient between the square of $R^{2}$ and the solution complexity, i.e. $\left(R^{2}\right)^{2} /$ complexity. Based on the overall score (0.063), the solution \#272 (whose tree is symbolically represented in Figure 8 ) was identified as the best GP formula for predicting $V^{*}$ and it was chosen for a last optimization process finalized at improving its generalization and at simplifying the weight coefficients.

Table 5 - Best performing solutions obtained for the formulation of $\gamma^{*}$ with GP.

\begin{tabular}{|c|c|c|c|c|}
\hline Solution ID & $\begin{array}{c}\text { Fitness score } \\
\left(\mathbf{R}^{2}\right)\end{array}$ & $\begin{array}{c}\text { Solution } \\
\text { complexity }\end{array}$ & $\begin{array}{c}\text { Overall score } \\
\left(\mathbf{R}^{2}\right)^{2} / \text { complexity }\end{array}$ & Solution \\
\hline 504 & 0.852 & 18 & 0.040 & $\begin{array}{c}0.003\left(\xi_{\mathrm{m}-1,0}-\varepsilon\right)-0.868 \tanh \left(\mathrm{h}_{w} / \mathrm{R}_{\mathrm{c}}\right)- \\
0.479 \cdot\left(\mathrm{B} / \mathrm{L}_{\mathrm{m}-1,0} \cdot \xi_{\mathrm{m}-1,0}\right)+0.044\left(\xi_{\mathrm{m}-1,0} \cdot \mathrm{h}_{\mathrm{w}} / \mathrm{R}_{\mathrm{c}}\right)+ \\
1.000\end{array}$ \\
\hline 621 & 0.850 & 14 & 0.052 & $\begin{array}{c}0.049 \cdot\left(\xi_{\mathrm{m}-1,0} \cdot \mathrm{h}_{\mathrm{w}} / \mathrm{R}_{\mathrm{c}}\right)-0.912 \tanh \left(\mathrm{h}_{\mathrm{w}} / \mathrm{R}_{\mathrm{c}}\right)- \\
0.480 \cdot\left(\mathrm{B} / \mathrm{L}_{\mathrm{m}-1,0} \xi_{\mathrm{m}-1,0)}\right)-0.003 \varepsilon+1.020\end{array}$ \\
\hline 272 & 0.834 & 11 & 0.063 & $\begin{array}{c}0.003\left(\xi_{\mathrm{m}-1,0}-\varepsilon\right)-0.300 \tanh \left(\mathrm{B} / \mathrm{L}_{\mathrm{m}-1,0)-}-\right. \\
0.495 \tanh \left(\mathrm{h}_{\mathrm{w}} / \mathrm{R}_{\mathrm{c}}\right)+0.995\end{array}$ \\
\hline 280 & 0.705 & 8 & 0.062 & $\begin{array}{c}0.998-0.616 \tanh \left(\mathrm{h}_{\mathrm{w}} / \mathrm{R}_{\mathrm{c}}\right)- \\
0.020 \tanh \left(\mathrm{B} / \mathrm{L}_{\mathrm{m}-1,0}\right)\end{array}$ \\
\hline
\end{tabular}

\subsection{Advantages and limits of GP}

One of the main advantages offered by GP (with respect to other machine-learning tools, such as neural networks) is the possibility to obtain a formula, instead of a black-box predicting model, with a relatively small number of parameters. A formula can be checked, corrected, updated and re-calibrated in case new data become available. Differently from neural networks, the human supervision and modification of the GP solutions is possible and desirable. Indeed GP belongs to the category of "supervised machine learning tools", where the data training process is supposed to get a feedback from the human experience.

In order to keep the predicting model simple and limit the number of freedom degrees, it may happen that even the "best performing" solution obtained with the application of GP is not fully satisfactory. Figure 10 illustrates the results of the application of solution \#272 of Table 5 to the prediction of $q$ for the datasets UB and VD. The predictions $\left(q_{G P, 272}\right)$ are obtained by including $V^{*}$ as modeled by solution \#272 in the EurOtop (2018) equations for $q$. The distribution of the normalized errors $\left(q_{G P, 272}-q_{\text {meas }}\right)$ as functions of $\xi_{m-1,0}$ shows a "clustering" of the larger errors around $\xi_{m-1,0}=2$. In case of non-breaking waves, the error decreases with $\xi_{m-1,0}$, while in case of breaking data are, the errors increase with $\xi_{m-1,0}$ up to $\xi_{m-1,0}=2$.

This analysis indicates that the formulation for $V^{*}$ by solution \#272 is not fully satisfactory, even if it represents the "best compromise" among the ones obtained with GP. One possibility would be searching another, more refined GP model than Solution \#272. This could be done by increasing the tree depth and the level of complexity of the mathematical relationships between the parameters. However, increasing the complexity means increasing the number of the 
coefficients and the freedom degrees of the solution, which may significantly compromise the "capability of generalization" of the solution itself. GP may deliver a more refined model for $\gamma^{*}$, but such model might result "too costumed" to fit the training data and might fail in the representation of new data (which is a typical shortcoming of other machine learning tools like neural networks, see Formentin and Zanuttigh, 2018b). In order to keep the model simple, Solution \#272 was selected and further elaborated as it is described in the following Sub-section 4.4 .
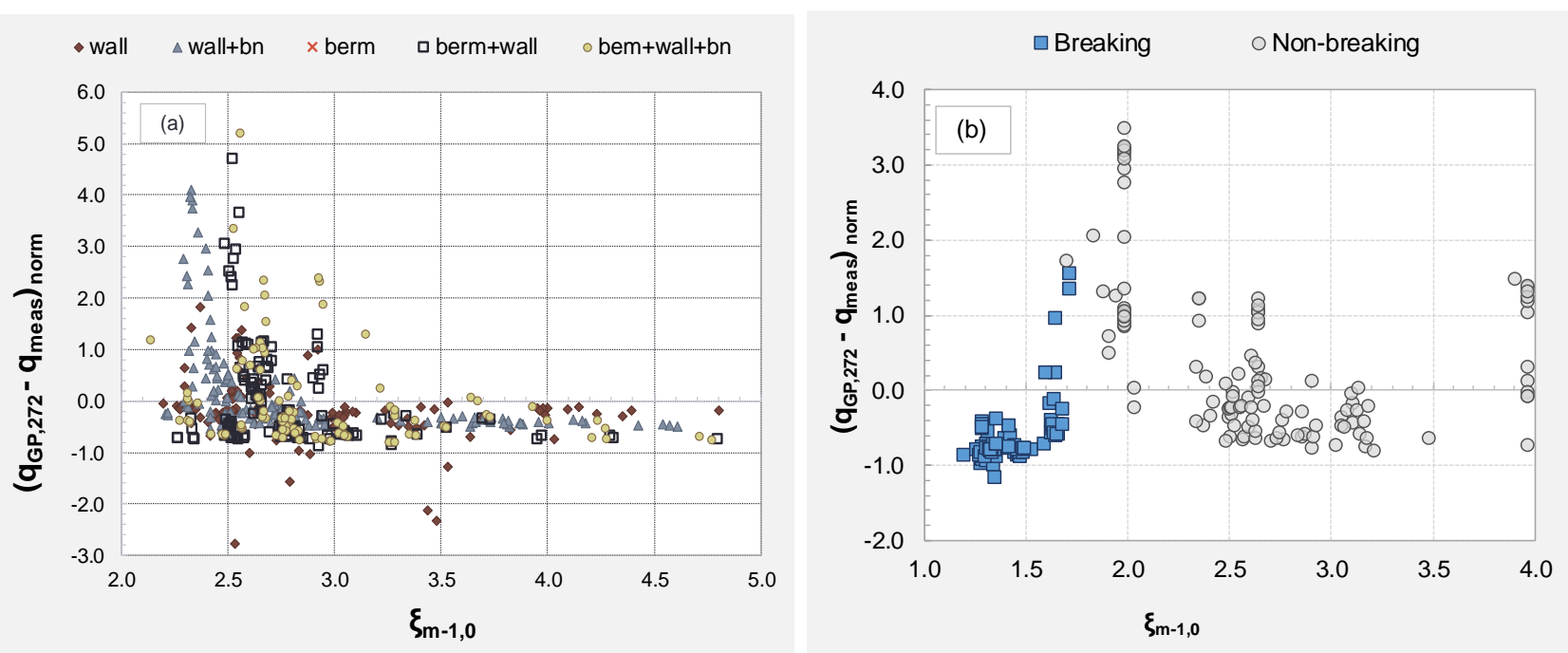

Figure 10 - Normalized errors between measurements $\left(q_{\text {meas }}\right)$ and predictions of $q$ obtained from the application of $V^{*}$ as modeled by solution \#272 $\left(q_{G P, 272}\right)$. The errors are plotted as functions of $\xi_{m-1,0}$. Panel a: VD-tests; panel b: UB-tests.

\subsection{The new formula}

The formula proposed in this contribution for the prediction of $V^{*}$ is therefore the result of the human supervised manipulation of solution \#272 of Table 5 . The formula is provided in the following Eq. (11):

$Y_{G P}^{*}=\left(\frac{0.93}{\tanh \left(1.5 \cdot \xi_{m-1,0}\right)}\right)-\left(0.30 \cdot \tanh \left(\frac{B}{L_{m-1,0}}\right)\right)-\left(0.40 \cdot \tanh \left(\frac{h_{w}}{R_{c}}\right)\right) \cdot-\left(0.15 \cdot \varepsilon_{r a d}\right)$,

where the subscript "GP" is added to distinguish the new $V^{\star}$ factor from the one proposed by VD, while the subscript "rad" indicates that the bn inclination angle $\varepsilon$ should be provided in radians. The formulation of Eq. (11) keeps the same form, the same parameters and the same signs of the solution \#272. It also involves the same hyperbolic tangent function (tanh) for $B / L_{m-1,0}$ and for $h_{w} / R_{c}$ and similar weights for $B / L_{m-1,0}, h_{w} / R_{c}$ and $\varepsilon$ (the numerical difference between the weights of $\varepsilon$ being due to the difference between radians and degrees). The use of tanh is particularly suitable to account for the reduction effects of the berm width and the wall height, as this function does not diverge, but converges to a constant for large values of $B / L_{m-1,0}$ and $h_{w} / R_{c}$. A linear 
function of $\varepsilon$ is instead sufficient as $\varepsilon$ spaces between 0 and $\pi / 2$, i.e. it cannot assume indefinitely high values.

385 With respect to the solution \#272, the main novelty of Eq. (11) is that $\gamma^{*}$ is a function of $386\left(\tanh \left(\xi_{m-1,0}\right)\right)^{-1}$ instead of increasing linearly with $\xi_{m-1,0}$. This change was made because the solution \#272 tended to give overestimations of $q$ for values of $\xi_{m-1,0}>2$. Indeed:

- $\quad V^{*}$ is independent of $\xi_{m-1,0}$ in case of non-breaking waves $\left(\xi_{m-1,0}>2\right)$, as already found by VD and confirmed by Zanuttigh and Formentin (2018);

- the effect $\xi_{m-1,0}$ is non-negligible in case of breaking waves $\left(\xi_{m-1,0} \leq 2\right)$ and it plays a positive contribution on $\gamma^{*}($ sign +$)$, differently from the other parameters $\left(B / L_{m-1,0}, h_{w} / R_{c}\right.$ and $\left.\varepsilon\right)$ which contribute to reduce $V^{*}$ (sign -), see Zanuttigh and Formentin (2018);

- the positive contribution of $\gamma^{*}$ should decrease with increasing $\xi_{m-1,0}$ up to approximately $\xi_{m-1,0}=2$, i.e. exactly when the boundary between breaking and non-breaking waves is reached.

The use of the function (tanh $\left.\left(1.5 \cdot \xi_{m-1,0}\right)\right)^{-1}$ introduces a positive contribution in the calculation of $V^{*}$ which monotonically decreases to 1 for increasing $\xi_{m-1,0}$. In the practice, for $\xi_{m-1,0}>2$, $\left(\tanh \left(1.5 \cdot \xi_{m-1,0}\right)\right)^{-1} \approx 1$. The bias term of the solution \#272 $(+0.995)$ is incorporated in the coefficient 0.93 multiplying $\left(\tanh \left(1.5 \xi_{m-1,0}\right)\right)^{-1}$. Ultimately, when $\xi_{m-1,0}>2$ the term $+0.93\left(\tanh \left(1.5 \cdot \xi_{m-1,0}\right)\right)^{-1}$ tends to +0.92 , i.e. a constant replacing the bias +0.995 of the solution \#272.

The main advantages of using Eq. (11) instead of the existing formulations from the literature 402 Eq. (1), Eqs. (4) and (6) are the following.

403

- For each of the structure types of Figure 8, Eq. (11) directly provides the $V_{G P}^{*}$ coefficient to 405 be included in the EurOtop (2018) formulae independently of the structure configuration. Indeed, when a structural element is not present, its corresponding term is equal to 0 (e.g.,

412 The new GP-based method consists therefore of the following Eqs.: if the structure has no berm, the term $0.3 \cdot \tanh \left(B / L_{m-1,0}\right)=0$. On the contrary, the application of the VD formulae requires to use a different formulation for each combination of structure elements (e.g., wall+berm, or wall+berm+bn, or wall+bn, etc.).

- Eq. (11) can be applied to both breaking and non-breaking waves.

- Eq. (11) allows a cautious approach, giving on average higher estimations of $q$ (see the next Section 5).

$\frac{q_{G P}}{\sqrt{g H_{m 0}^{3}}}=\frac{0.023}{\sqrt{\tan \alpha_{d}}} \cdot \xi_{m-1,0} \cdot \exp \left(-\left(2.7 \cdot \frac{R_{c}}{\xi_{m-1,0} \cdot H_{m 0} \cdot V_{G P}^{*}}\right)^{1.3}\right), \quad \xi_{m-1,0} \leq 2$

415 where $Y_{G P}^{*}$ is given by Eq. (11). Eqs. (11) and (12) will be named the "GP method" in the following 


\section{Results and verification of the new formula}

419 This Section presents the results of the application of the GP method to the new database of 420 numerical and experimental data (Sub-section 5.1) and to the database by VD (Sub-section 5.2). 421 For each dataset, the predictions of $q_{G P}$ were compared to the corresponding measurements $422\left(q_{\text {meas }}\right)$ and to the predictions obtained with the literature methods ( $q_{V D}$ by Eq. 1 and $q_{Z F}$ by Eq. 423 7). The performance of the new formula is quantitatively assessed and compared to the 424 performance of the existing methods by means of the indexes $R^{2}$ and SK. The values of these 425 indexes are reported in Table 3 for each dataset and for each predicting method where 426 applicable. Graphical charts are also included to give a qualitative idea of the performance of 427 the new formula. Figures 11 and 13a illustrate the distribution of $q_{G P}$ vs $q_{\text {meas }}$, while Figures 12 428 and $13 \mathrm{~b}$ show the distribution of the $q_{\text {meas }}$-values around the curves representing Eqs. (12a) and 429 (12b).

\subsection{Application to the new database}

431 The new GP method by Eq. (12) is here tested against the UB-data. Overall, the level of 432 uncertainty associated to the prediction of the whole set of new data by $q_{G P}$ is represented by a 433 percentage standard deviation $\sigma_{\%}$ of $10.0 \%$, varying between $9.0 \%$ and $17 \%$ for the experimental 434 and numerical data.

435 The quantitative and the qualitative analyses of the results are given respectively in Table 3 and 436 in Figures 11 and 12. The aim is to characterize the performance of the new formula on the 437 whole database and on sub-datasets of tests grouped by: i) experimental and numerical tests; 438 ii) breaking and non-breaking waves; iii) smooth berms with walls including or not a bn.

439 For the whole database and for each groups of tests, the values of $R^{2}$ and $S K$ are presented in 440 the upper part of Table 3, in comparison to the same performance indexes obtained with the 441 literature methods, i.e. VD, Eur 2018 and ZF.

442 For each dataset and on the whole database, the use of $\gamma_{G P}^{*}$ determines an improvement of the 443 performance with respect to the use of $y^{*}$ and $y^{* *}$, both in terms of accuracy $\left(\mathrm{R}^{2}\right)$ and of symmetry 444 (SK). The best accuracy is achieved on the experimental dataset $\left(R^{2}=0.908\right)$, the breaking tests $445 \quad\left(R^{2}=0.922\right)$ and the structures without bns $\left(R^{2}=0.906\right)$. The lowest values of $R^{2}$ are observed on 446 the datasets of numerical tests $\left(R^{2}=0.760\right)$, non-breaking waves $\left(R^{2}=0.764\right)$ and structures with 447 bns $\left(R^{2}=0.779\right)$. This slightly lower performance is principally determined by the inherent 448 uncertainty associated to the smallest $q$-values measured in the lab and in the numerical model 449 (indicatively, $q<10^{-5} \mathrm{~m}^{3} /(\mathrm{sm})$ ), especially in presence of structures with bns. In case of 450 (extremely) rare overtopping, indeed, the overtopping volumes collected in the numerical 451 reservoir and in the laboratory tank (see Figures 2 and 4) might be so small to be close to the 452 mesh resolution or to the precision of the hydrometer. Anyway, even in such conditions, the accuracy 453 of the predictions of $\gamma_{G P}^{*}$ is still satisfactory $\left(R^{2}>0.75\right)$, especially if compared to the value of $R^{2} \approx 0.68$ 454 obtained for these datasets with ZF. 
The most important achievement obtained with the new formula is represented by the SK values. The results of Table 3 remark that the predictions of $q$ obtained with all the other methods are systematically affected by an underestimation bias, no matter which dataset they are applied on. The corresponding values of $S K$ are all negative and relatively high $(-1.95 \leq S K \leq-0.91)$, if considering the absolute values. On the contrary, the $S K$ values associated to $Y_{G P}^{*}$ are all positive but one (structures with berm, wall and bns, $S K=-0.26)$ and lower in magnitude $(-0.26 \leq S K \leq 1.50)$. This means that the new formula gives more conservative and less biased estimations of $q$, outperforming the existing methods which tend to systematically underestimate the $q$-values.

463 From a qualitative viewpoint, the 2 charts of Figure 11 display the same distribution $q_{G P}$ vs $q_{\text {meas }}$ by grouping the data into breaking and non-breaking waves (panel a) and into structures with and without bn (panel b). The distinction between structures with and without bn is also proposed in Figure 12, which compares the measurements $q_{\text {meas }} /\left(\mathrm{gH}^{3}{ }_{\mathrm{mo}}\right)^{0.5}$, as functions of $R_{d} / H_{m 0}$, to the curve representing the EurOtop (2018) formulae with $V_{G P}^{*}$ for breaking and non-breaking waves in panel $a$ and $b$, respectively. Figure 11 shows that the predictions are almost symmetrically distributed around the bisector line and are not affected by any particular bias or heteroscedasticity related to any of the 4 groups of tests. In Figure $12 \mathrm{a}$, all the breaking tests are aligned around the fitting line by Eq. (12a) and included within the $90 \%$ confidence bands. In Figure (12b), the non-breaking tests tend to fall around or slightly below the curve of Eq. (12b), suggesting that - especially in case of structures without bns - the new formula tends to give cautious estimations of $q$.

The application of the new factor $V_{G P}^{*}$ to the new database gives satisfactory results, considering the agreement among predictions and measurements, the symmetry of the error distribution and the agreement with the curves representing the formulae. Further improvements might be achieved by focusing on a more detailed representation of the rare overtopping conditions.
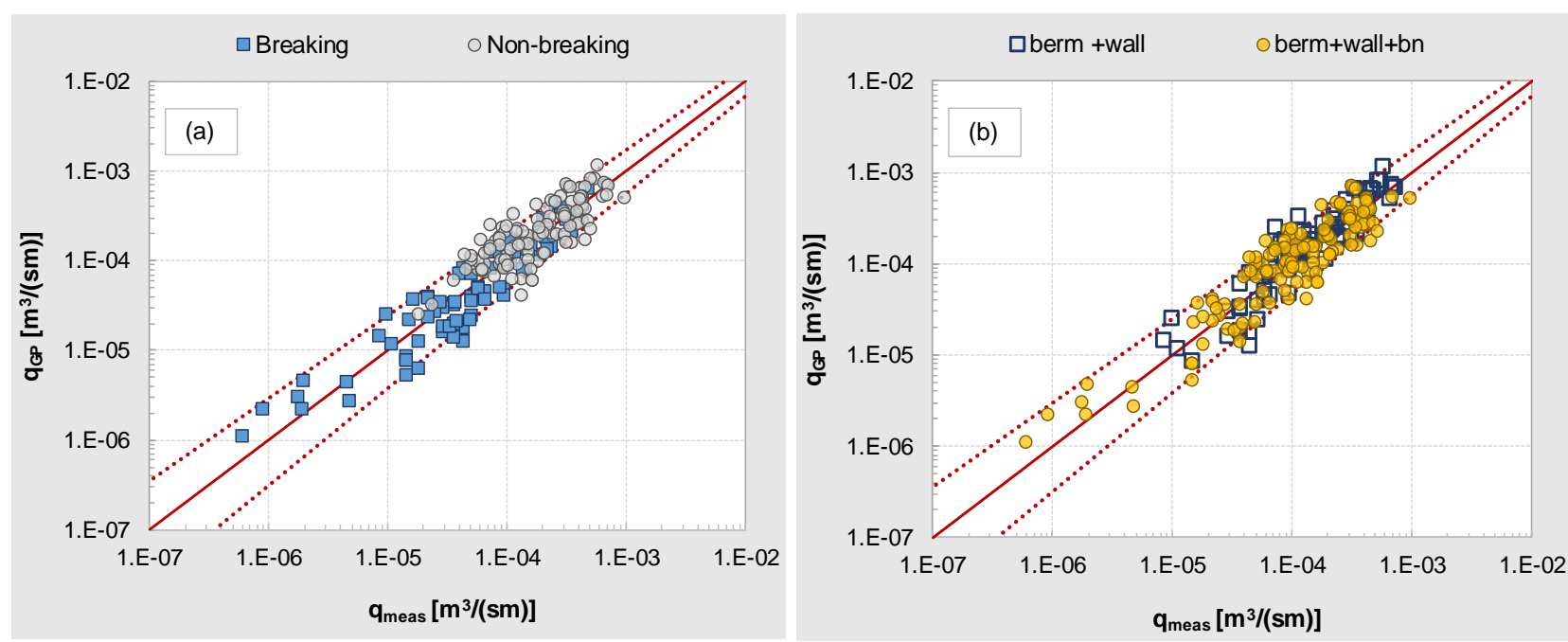

Figure 11 - Predictions of $q\left(q_{G P}\right)$ obtained with the new GP method (Eq. 12) compared to the 483 corresponding measurements $\left(q_{\text {meas }}\right)$. The tests belong to the UB-dataset and are distinguished by breaking and non-breaking conditions in panel (a) and by structures with berm and wall and 
structure with berm, walls and bns in panel (b). The dotted lines represent the $90 \%$ confidence bands associated to the predictions.
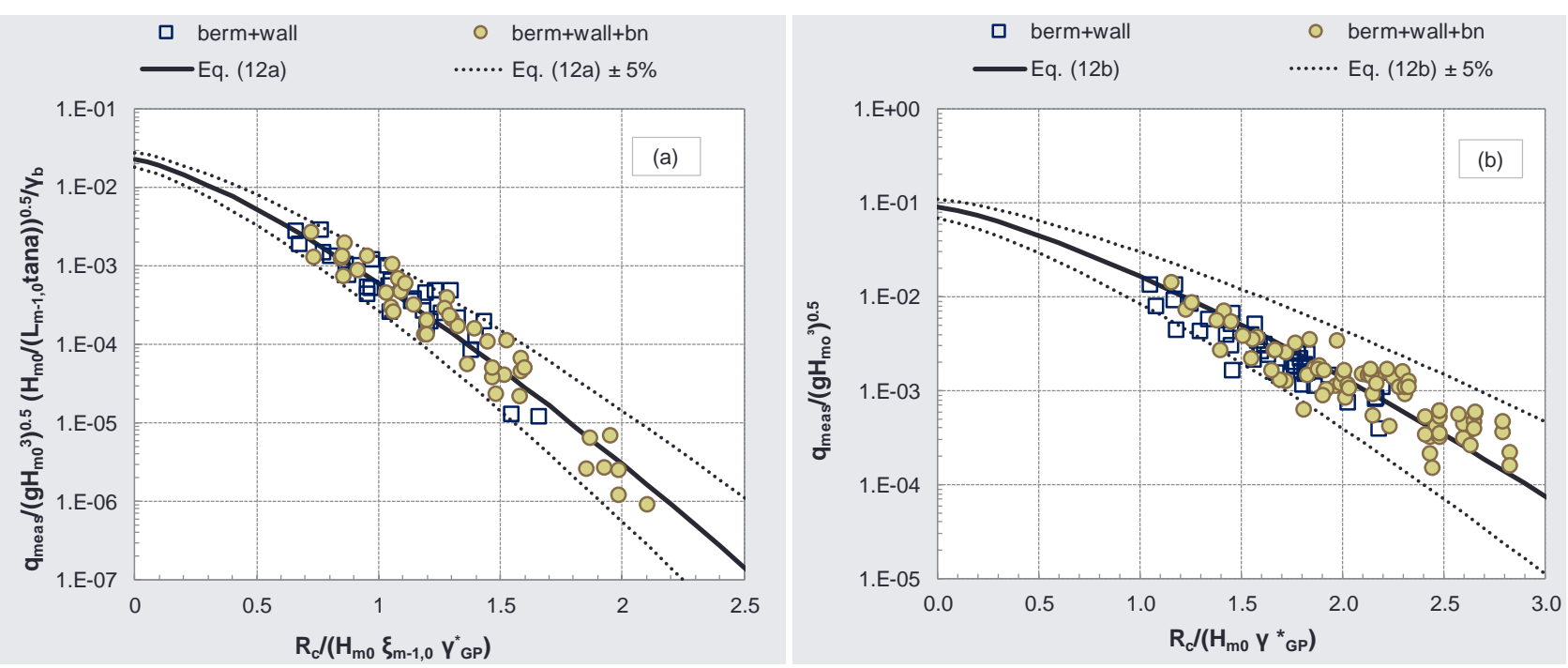

Figure 12 - Dimensionless discharges $q_{\text {meas }} /\left(\mathrm{gH}^{3}{ }_{\mathrm{mo}}\right)^{0.5}$ from UB-data as functions of $R_{d} H_{m 0}$ compared to the curve (continuous line) representing the GP method in case of breaking (panel a, Eq. 12a) and non-breaking (panel b, Eq. 12b) conditions. The dotted lines are to the $90 \%$ confidence bands.

\subsection{Application to the database by VD}

The quantitative results of the application of the GP method to the VD dataset are reported in the lower part of Table 3. The performance indexes $\mathrm{R}^{2}$ and SK were calculated for $q_{G P,} q_{V D}$, $q_{E u r, 2018}$ and $q_{Z F}$ on the whole VD database and on 5 datasets of tests grouped according to the structural complexity: i) wall, ii) wall and bn, iii) berm, iv) berm and wall, v) berm, wall and bn. The average level of uncertainty associated to the prediction of the whole VD-database by $q_{G P}$ is represented by $\sigma_{\%}=6.1 \%$, with $\sigma_{\%}=3.6 \%$ on the dataset with wall and bns and $\sigma_{\%}=9.3 \%$ on the dataset with berm, wall and bn.

The $\mathrm{R}^{2}$ values obtained with $q_{E u r, 2018}$ and $q_{Z F}$ are almost identical, because the formulation of $q_{Z F}$ converge to the formulations of $q_{E u r, 2018}$ in case of non-breaking waves. The differences in the $\mathrm{R}^{2}$ values between $q_{G P}$ and $q_{E u r, 2018}$ or $q_{Z F}$ all refer to the $3^{\text {rd }}$ significant figure and therefore can be considered negligible in the practice. The $\mathrm{R}^{2}$ values associated to $q_{V D}$ are slightly higher, and the differences with $q_{G P}$ reach the $2^{\text {nd }}$ significant figure for the dataset on berm+wall+bn. Indeed, $q_{V D}$ was specifically developed on these data and is therefore characterized by the greatest accuracy.

Similarly to the application on the new database, the substantial improvement related to the use of $V_{G P}^{*}$ is represented by the SK values. With the exception of the datasets of berms $(S K=-1.12)$ and berms+walls+bns $(S K=-0.15)$, the predictions of $q_{G P}$ are all characterized by positive and on average lower values of $S K$ in absolute terms $(-1.12 \leq S K \leq 2.55)$, than the predictions obtained 
with $q_{E u r, 2018}(-2.24 \leq S K-0.97)$ and $q_{Z F}(-2.21 \leq S K \leq-1.12)$. This means that the errors $\left(q_{G P}-q_{\text {meas }}\right)$ are generally more symmetrically distributed around 0 and are more frequently positive, i.e. $q_{G P}>q_{\text {meas }}$. On the contrary, $q_{E u r, 2018}$ and $q_{Z F}$ systematically underestimate all the data, as it can be appreciated in Figure 7. The values of SK obtained by $q_{V D}$ are comparable to the ones by $q_{G P}$ $(-2.03 \leq S K \leq 2.38)$ confirming that the original method proposed by VD is accurate and conservative in case of non-breaking waves.

The quantitative results are reflected in the 2 plots of Figure 13: panel (a) shows the distribution of $q_{G P}$ vs $q_{\text {meas }}$, while panel (b) provides the distribution of the measured values $q_{\text {meas }} /\left(\mathrm{gH}_{\mathrm{mo}}\right)^{0.5}$ vs $R_{d}\left(H_{\left.m o V_{G P}^{*}\right)}\right.$ in comparison to EurOtop (2018). Figure 13a and Figure 7 a display the same distributions of values, obtained with $q_{G P}$ and $q_{E u r, 2018}$, respectively. While almost all the predictions fall below the bisector line of Figure 7a, the data in Figure 13a are on average symmetrically distributed around the bisector line. In line with the SK-values of Table 3, the dataset most-symmetrically distributed in Figure $13 \mathrm{a}$ is the one on berms+wall+bn $(\mathrm{SK}=-0.15$, i.e. the lowest absolute value of SK), while the dataset on berms in the only one which tends to be slightly underestimated $(S K=-1.12)$. Figure $13 \mathrm{~b}$ shows that the predictions $q_{G P}$ are still consistently aligned along the curve of EurOtop (2018) (continuous line) and most of the data fall within the corresponding $90 \%$ confidence bands (dotted lines). Therefore, the introduction of $V_{G P}^{*}$ significantly reduces the underestimation bias associated to $q_{E u r, 2018}$ and $q_{Z F}$ and it is physically consistent with $R_{c} / H_{m o}$.
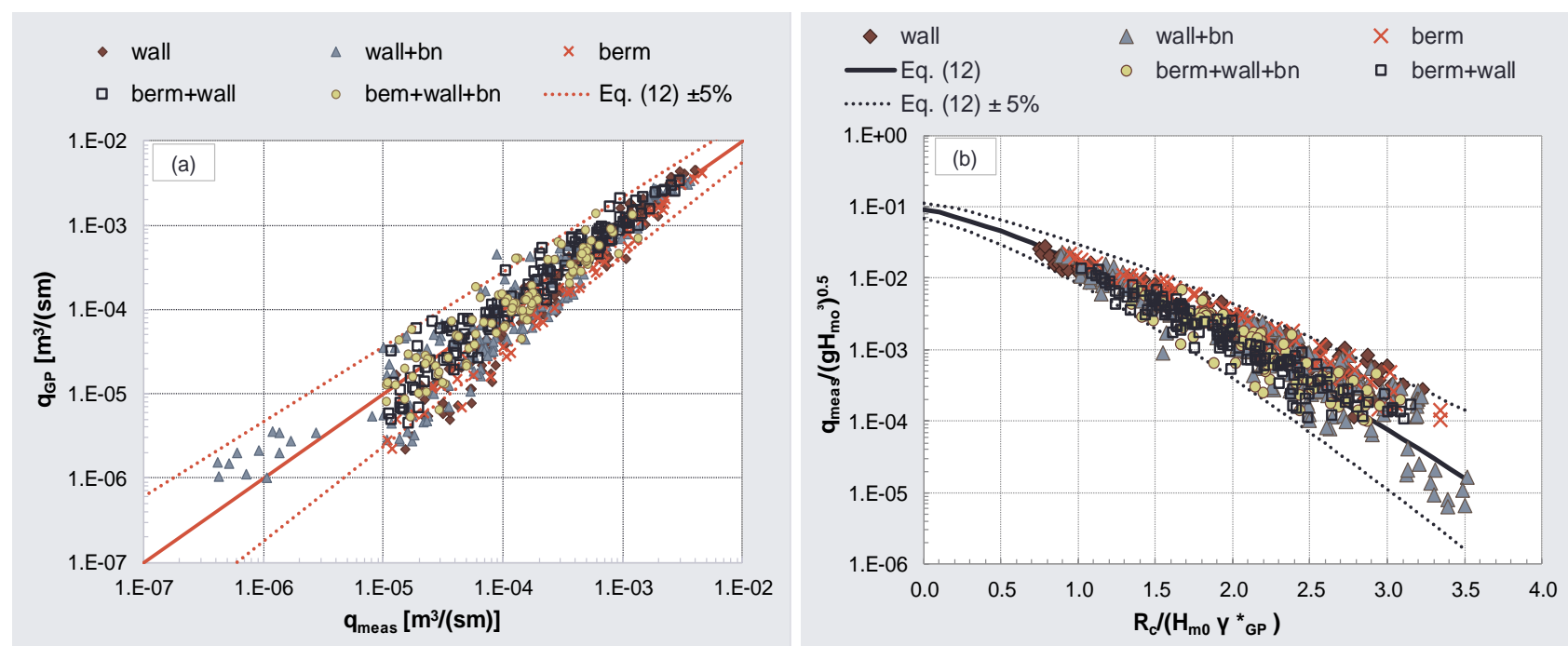

Figure 13 - Panel a: predictions of $q\left(q_{G P}\right)$ obtained with the new formula for $V_{G P}^{*}$ (Eq. 11) compared to the corresponding measurements $\left(q_{\text {meas }}\right)$. Panel b: dimensionless measurements $\mathrm{q} /\left(\mathrm{gH}^{3}{ }_{\mathrm{mo}}\right)^{0.5}$ as functions of $R_{d} / H_{m 0}$ compared to the curve representing the EurOtop (2018) formulae for the prediction of $q$ in case of non-breaking wave conditions. The data belong to the VD-dataset. The dotted lines refer to the $90 \%$ confidence bands. 


\section{Conclusions}

540 This contribution proposed a new coefficient $\gamma_{G P}^{*}$ to represent the effects of crown walls and 541 bullnoses (bns) on the reduction of $q$. The coefficient is conceived to be included in the EurOtop 542 (2018) formulae for the prediction $q$ : it is supposed to replace the coefficient $V^{*}$ in the non543 breaking waves formula and the coefficients $\gamma_{v}$ and $\gamma_{b}$ in the formula for breaking waves.

544 The new formula for calculating $V_{G P}^{*}$ has been developed by applying the Genetic Programming (GP) technique to a set of existing and new data on wave overtopping at composite structures with bns. These data essentially consist of the database collected by Van Doorslaer et al. (2015) and the new experimental and numerical database collected by the authors and presented in this contribution. The new experiments were conducted in the wave flume of the Laboratory of Hydraulics of the University of Bologna while the numerical simulations were carried out with the consolidated IH2VOF code developed by the University of Cantabria and validated against experimental data with bns. The new experiments and the new numerical modelling involved smooth berms, dikes with crown walls and bns under breaking and non-breaking wave conditions. The reliability of these new data was checked against the existing predicting methods (EurOtop, 2018).

A sensitivity analysis was carried out to individuate the most suitable set of parameters to be included in the GP training to describe the effects of the structural elements and of the wave breaking. The optimal set consisted of: the ratio between the berm width and the wave length $B / L_{m-1,0}$, the ratio between the wall height and the total structure freeboard $h_{w} / R_{c}$, the angle of inclination of the bn $\varepsilon$ and the breaker parameter $\xi_{m-1,0}$. The application of the GP prompted a number of similar formulations for calculating $V_{G P}^{*}$ as a function of these parameters.

The GP best performing formulation - i.e. the formulation that provides the values of $Y^{*} G P$ which allows the most accurate estimations of $q$ - was selected and optimized under the human supervision to obtain a physically-based and physically-coherent predicting method. The final result is a unique, practical formula for predicting $V_{G P}^{*}$ accounting for the single or contemporary presence in the dike layout of one or more structural elements (berm, wall and bn) through the simple sum of the corresponding parameters. The dependency of $V^{*} G P$ on each parameter is expressed through the hyperbolic tangent function, which is automatically equal to 0 when the element is absent and which is intrinsically upper-limited when the parameters tend to $\infty$.

The application of the new formula to the new data and the data by VD demonstrates that the use of $\gamma_{G P}^{*}$ in the EurOtop (2018) formulae allows to get estimations of $q$ that are at least as accurate as the existing methods and that vary consistently with the physical parameters. The accuracy is represented by values of the coefficient of determination $R^{2}$ computed among predictions and measurements of $q$ that are never lower than $\approx 0.76$ and in most cases $>0.85$. The main improvement of the new formula consists of the possibility to overcome the underestimations of $q$, which would be obtained using some of the recent literature methods. It was indeed verified that the application of the coefficient $V^{*}$ by VD - which was originally conceived to be included in the EurOtop (2007) formulae for $q$ - to EurOtop (2018), and its modified version $V^{* *}$ developed by Zanuttigh and Formentin (2018), lead to systematic 
underestimations of $q$ independently of the dataset and of the combination of structural elements of the dike configuration. The entity of the underestimations was quantified through the calculation of the skewness coefficient (SK) of the distribution of the differences between measurements and predictions of $q$ obtained with the different methods. For any dataset or group of data, the SK values associated to $\gamma^{*}$ and $\gamma^{* *}$ are systematically $<0$, while the SK values associated to $Y_{G P}^{*}$ are positive and significantly closer to 0 .

The possibility to directly apply the unique, new formula to the EurOtop (2018) equations for $q$ represents a remarkable improvement with respect to the method by VD, which consists of several different formulations (as reported in Appendix 1) to be selected each time according to the different combination of structural elements contemporary present (bullnose, promenade, crown wall).

The range of validity of the new formula is determined by the range of the parameters characterizing the data used to train the GP and to calibrate the coefficients of the formula itself, which are reported in Table 1. The formula has been applied so far to smooth dikes with crown walls under perpendicular waves only.

\section{Acknowledgments}

The authors would like to express their sincere gratitude to Dr. Koen van Doorslaer, for providing the experimental data, and to Dr. Jentsje W. van der Meer for the long-term cooperation.

The support of the European Commission through the Horizon 2020 project BRIGAID ("BRIdging the GAp for Innovations in Disaster resilience", www.brigaid.eu) is gratefully acknowledged.

\section{References}

Burcharth, H. F., Lykke Andersen 'T. and Lara, J.L., 2018. Upgrade of coastal defence structures against increased loadings caused by climate change: A first methodological approach, Coastal Engineering, 87, 112-121.

Cox, R.J and Tajziehchi, M., 2005. 2D experimental modelling of hydrodynamic effects of submerged breakwaters. Proceedings of 5th International Conference on Coastal Dynamics, Barcelona (S).

EurOtop, 2007. European Manual for the Assessment of Wave Overtopping. T. Pullen, N.W.H. Allsop, T. Bruce, A. Kortenhaus, H. Schüttrumpf and J.W. van der Meer.

EurOtop, 2018. Manual on wave overtopping of sea defences and related Structures. An overtopping manual largely based on European research, but for worldwide application. N.W.H. Allsop, T. Bruce, J. DeRouck, A. Kortenhaus, T. Pullen, H. Schüttrumpf, P. Troch, J.W. van der Meer and B. Zanuttigh. www.overtopping-manual.com 
613 Formentin, S.M., Zanuttigh, B., van der Meer, J.W. and Lara, J.L., 2014. Overtopping flow 614 characteristics at emerged and over-washed dikes, Proc. of XXXIV ICCE, Seoul (ROK).

615 Formentin S.M., Zanuttigh B. and J.W. van der Meer, 2017. A neural network for predicting wave 616 reflection, overtopping and transmission, Coastal Engineering Journal, 59, No. 2, 1750006, 31 pp.

617 Formentin, S.M. and Zanuttigh, B., 2018a. A new method to estimate the overtopping and 618 overflow discharge at over-washed and breached dikes, Coastal Engineering, 140, 240-256.

619 Formentin S.M. and Zanuttigh, B., 2018b. A methodological approach for the development and 620 verification of artificial neural networks based on an application to wave-structure interaction 621 processes, Coastal Engineering Journal 60(3), 260-279.

622 Galvin, C., 1964. Wave-height prediction for wave generators in shallow water. Technical memorandum n.4. March 1964, 21 p. U.S. Army Coastal Engineering Research Center.

Gaur, S. and Deo, M.C., 2008. Real-time wave forecasting using genetic programming, Ocean Engineering 35, 1166-1172.

Hughes, S. A., and Nadal, N. C., 2009. Laboratory study of combined wave overtopping and storm surge overflow of a levee, Coastal Engineering, 56(3), 244-259.

Kortenhaus, A., Haupt, R., Oumeraci, H., 2001. Design aspects of vertical walls with steep foreland slopes. Proceedings of ICE 2001, 221-232.

Kortenhaus, A., Pearson, J., Bruce, T., Allsop, W., van der Meer, J.W., 2003. Influence of parapets and recurves on wave overtopping and wave loading of complex vertical walls. Proceedings of Coastal Structures 2003.

Koza, J.R, 1992. Genetic Programming: On the Programming of Computers by Means of Natural Selection. MIT Press, Bradford Book.

Lara, J.L., Ruju, A. and Losada, I.J., 2011. Reynolds Averaged Navier-Stokes modelling of long waves induced by a transient wave group on a beach. Proceedings of the Royal Society A, vol. 467, 1215-1242.

Losada, I.J., Lara J.L., Christensen, E.D. and Garcia N., 2005. Modelling of velocity and turbulence fields around and within low-crested rubble-mound breakwaters, Coastal Engineering, 52(10-11), 887-913.

Liu, P.L.-F., Lin, P., Chang, K.-A., Sakakiyama, T., 1999. Numerical modelling of wave interaction with porous structures. J. Waterw., Port, Coast., and Ocean Eng., 125 (6), 322 - 330.

Muttray, M., Oumeraci, H. and Ten Oever, E., 2006. Wave Reflection and Wave Run-Up at Rubble Mound Breakwaters. Proc. of XXX ICCE, San Diego (CA). 
Nr. 860, Technishe Universität Braunschweig, Leichtweiß-Institut für Wasserbau, Abteilung Hydromechanik und Küsteningenieurwesen (in German).

Owen, M.W. and Steele, A.A.J., 1991. Effectiveness of Recurved Wave Return Walls, HR Wallingford, Report SR 261.

Pilechi A., Baker S. and Cornett A., 2018. Evaluation of a numerical wave modelling tool for studying the overtopping of rubble mound breakwaters, Proceedings of VII International Conference on the Application of Physical Modelling in Coastal and Port Engineering and Science (Coastlab18), Santander (S).

Pourzangbar, A., Losada M.A., Saberc A., Rasoul Aharid, L., Larroudé, P., Vaezif, M. and Brocchini, M., 2017. Prediction of non-breaking wave induced scour depth at the trunk section of breakwaters using Genetic Programming and Artificial Neural Networks, Coastal Engineering 121, 107-118.

Power, H.E., Gharabaghi, B., Bonakdari, H.,, Atkinsond, A.L. and Baldock, T.E., 2019. Prediction of wave runup on beaches using Gene-Expression Programming and empirical relationships, Coastal Engineering, 144, 47-61.

Searson, D., 2009. GPTIPS: Genetic Programming \& Symbolic Regression for MATLAB, http://gptips.sourceforge.net.

Van der Meer, J.W and Bruce, T., 2014. New Physical Insights and Design Formulas on Wave Overtopping at Sloping and Vertical Structures, Journal of Waterway, Port, Coastal, and Ocean Engineering, 140.

Van Doorslaer, K., De Rouck, J., Audenaert, S. and Duquet, V., 2015. Crest modifications to reduce wave overtopping of non-breaking waves over a smooth dike slope, Coastal Engineering, $101,69-88$.

Wang, S., 1974. Plunger-Type Wave Makers: Theory and Experiment. J. of Hydraulic Research, 12(3), $357-388$.

Zanuttigh B., Zagonari F., Bagli S., Pescaroli G., Bozzeda F., Nicholls R., Hoggart S. and Vanderlinden, J-P., 2014. Theseus decision support system for coastal risk management, Coastal Engineering, 87, 218-239.

Zanuttigh B., Formentin S.M., and van der Meer., J.W., 2016. Prediction of extreme and tolerable wave overtopping discharges through an advanced neural network, Ocean Engineering, 127, 7-22.

Zanuttigh B. and Formentin S.M., 2018. Reduction of the wave overtopping discharge at dikes in presence of crown walls with bullnoses, Proc. of XXXVI ICCE, Baltimore (MD). https://doi.org/10.9753/icce.v36.papers. 110

Zelt, J.A., Skjelbreia, J.E., 1992. Estimating incident and reflected wave field using an arbitrary number of wave gauges. Proc. of XXIII ICCE 1992, Venice (I) vol I, 777-789. 


\section{Appendix 1. Formulations for $Y^{*}$ by Van Doorslaer et al. (2015)}

682 The formulations by Van Doorslaer et al. (2015) for the parametrization of the coefficient $V^{*}$ to 683 be included in EurOtop (2007) to predict $q$ in case of non-breaking waves $\left(\xi_{m-1,0}\right)$ correspond to 684 EurOtop Eqs. 5.45-5.50 and are reported in the following in relation to structure configuration.

685 - smooth dike + wall:

686

$$
V^{*}=\gamma_{v}=\exp \left(-0.56 \cdot \frac{h_{w}}{R_{c}}\right), \quad \text { for } \frac{h_{w}}{R_{c}}=0.08-1.00
$$

- smooth dike + wall + bn:

689

$$
\gamma^{*}=\gamma_{v} \cdot \gamma_{b n} \cdot \gamma_{s 0, b n}, \quad \text { where: }
$$

$$
\text { ○ } V_{b n}=1.8 \gamma_{\varepsilon} \gamma_{\lambda} \text {, for } \frac{h_{w}}{R_{c}} \geq 0.25 \text {, where }
$$

690

$$
V_{\varepsilon}=\left\{\begin{array}{c}
1.53 \cdot 10^{-4} \varepsilon^{2}-1.63 \cdot 10^{-2} \varepsilon+1 \text { if } 15^{\circ} \leq \varepsilon \leq 50^{\circ} \\
0.56, \text { if } \varepsilon>50^{\circ}
\end{array}\right.
$$

and

$$
V_{\lambda}=0.75-0.20 \lambda \text {, if } 0.125 \leq \lambda \leq 0.6
$$

○ $\gamma_{b n}=1.8 \gamma_{\varepsilon} \gamma_{\lambda}-0.53, \quad$ for $\frac{h_{w}}{R_{c}}<0.25$, where

695

696

$$
V_{\varepsilon}=1-0.003 \varepsilon \quad \text { and }
$$$$
V_{\lambda}=1-0.144 \lambda \text {, }
$$

$$
\text { if } 0.1 \leq \lambda \leq 1
$$

- smooth dike + berm (or promenade):

$$
V^{*}=\gamma_{\text {prom }}=1-0.47 \frac{B}{L_{m-1,0}}, \quad \text { for } \frac{B}{L_{m-1,0}}=0.05-0.5
$$

- smooth dike + berm + wall:

$$
V^{*}=\gamma_{\text {prom }-v}=0.87 \cdot v_{\text {prom }} \cdot v_{v}, \quad \text { for } \frac{\mathrm{B}}{\mathrm{L}_{\mathrm{m}-1,0}}=0.05-0.4 \text { and } \frac{h_{w}}{R_{c}}=0.07-0.80 \text {, }
$$

699 with $\gamma_{v}$ from Eq. (A1) and $\gamma_{\text {prom }}$ from Eq. (A3)

700 - smooth dike + berm + wall + bn:

701

$$
v^{*}=\gamma_{\text {prom_v } v b n}=1.19 \cdot \gamma_{\text {prom_v }} \cdot \gamma_{b n}
$$

702

$$
\text { for } \frac{B}{L_{m-1,0}}=0.04-0.4, \frac{h_{w}}{R_{c}}=0.17-0.80, \varepsilon=30^{\circ}, 45^{\circ} \text { and } \lambda=0.25-0.38
$$

with $Y_{\text {prom }} v$ from Eq. (A4) and $\gamma_{b n}$ from Eq. (A2a) or (A2b) 


\section{Appendix 2. GP glossary}

706 The main terms and concepts characterizing the GP technique are listed and briefly described in the following. A schematic representation is also given in Figure 8.

- Tree: symbolic and conceptual representation of the computer program (formula) evolved by the GP;

- individual: solution (i.e. mathematical formula) to the task to be solved;

- population: set of individuals of the same generation (i.e. iteration);

- genes: the parameters, or variables, characterizing an individual (i.e. each term composing a solution formula);

- selection phase: phase of the algorithm in which the fittest individuals are selected to pass their genes to the next generation, i.e. to reproduce themselves;

- training data: set of empirical input values and corresponding output values from the process to be modelled;

- testing data: another set of input and corresponding output values that can be used, at the end of the run of GP, to evaluate the evolved models. The testing data are not used to evolve the models, but gives an indication of how well the models performs on new data not used for training (i.e. how well the models generalize).

- fitness function: function or criterion used to assess the "fitness" of each individual of a population to the task (e.g. the RMSE criterion); the fitness function gives a fitness score to each individual. The higher the fitness score, the higher the probability for an individual to be selected for "reproduction";

- parents: couple of individuals that are selected for reproduction on the basis of their fitness scores;

- cross-over: phase of the GP where the genes of the parents are randomly exchanged to create a new off-spring individual; the genes of the off-spring individual are thus a combination of the genes of the parents;

- mutation: like in nature, during the cross-over, there is a low-probability that a random mutation occurs to the off-spring genes; when this happens, some of the genes of the offspring can be flipped. The mutation is included in the GP to keep the population diversity and prevent premature convergences of the algorithm (i.e. to prevent that the fitness function falls entrapped in local minima);

- termination criterion: when the fitness function has reached its minimum or when the population does not produce any significant improvement with the next generation, the termination criterion is satisfied and the algorithm interrupts. 